\title{
RIGIDITY PHENOMENA IN MANIFOLDS WITH BOUNDARY UNDER A LOWER WEIGHTED RICCI CURVATURE BOUND
}

\author{
YOHEI SAKURAI
}

\begin{abstract}
We study Riemannian manifolds with boundary under a lower $N$-weighted Ricci curvature bound for $N$ at most 1 , and under a lower weighted mean curvature bound for the boundary. We examine rigidity phenomena in such manifolds with boundary. We conclude a volume growth rigidity theorem for the metric neighborhoods of the boundaries, and various splitting theorems. We also obtain rigidity theorems for the smallest Dirichlet eigenvalues for the weighted $p$-Laplacians.
\end{abstract}

\section{INTRODUCTION}

In this paper, we study Riemannian manifolds with boundary under a lower weighted Ricci curvature bound, and under a lower weighted mean curvature bound for the boundary. We develop the preceding studies of the author [33]. As explained below, we examine rigidity phenomena in such manifolds with boundary beyond the usual weighted setting.

For $n \geq 2$, let $M$ be an $n$-dimensional Riemannian manifold with or without boundary with Riemannian metric $g$, and let $f: M \rightarrow \mathbb{R}$ be a smooth function. We denote by $\mathrm{Ric}_{g}$ the Ricci curvature defined by $g$, by $\nabla f$ the gradient of $f$, and by Hess $f$ the Hessian of $f$. For $N \in(-\infty, \infty]$, the $N$-weighted Ricci curvature $\operatorname{Ric}_{f}^{N}$ is defined as

$$
\operatorname{Ric}_{f}^{N}:=\operatorname{Ric}_{g}+\operatorname{Hess} f-\frac{\nabla f \otimes \nabla f}{N-n}
$$

if $N \in(-\infty, \infty) \backslash\{n\}$; otherwise, if $N=\infty$, then $\operatorname{Ric}_{f}^{N}:=\operatorname{Ric}_{g}+\operatorname{Hess} f$; if $N=n$, and if $f$ is a constant function, then $\operatorname{Ric}_{f}^{N}:=\operatorname{Ric}_{g}$; if $N=n$, and if $f$ is not constant, then $\operatorname{Ric}_{f}^{N}:=-\infty$ ([2]). We notice that the parameter $N$ has been usually chosen from $[n, \infty]$.

Date: April 6, 2017.

2010 Mathematics Subject Classification. Primary 53C20.

Key words and phrases. Manifold with boundary; Weighted Ricci curvature.

Research Fellow of Japan Society for the Promotion of Science for 2014-2016. 
On manifolds without boundary under a lower $N$-weighted Ricci curvature bound, many results have been already known in the usual weighted case of $N \in[n, \infty]$ (see e.g., [20], 21], 22], 30, 34], 35], [38]). Recently, in the complemental weighted case of $N \in(-\infty, n)$, several geometric properties have begun to be studied (see [16], [17], [23], [25], [26], [27], [40]). Wylie [39] has obtained a splitting theorem of Cheeger-Gromoll type (cf. [8]) in the complementary weighted case of $N \in(-\infty, 1]$, and asked a question whether the splitting theorem can be extended to the remaining case of $N \in(1, n)$.

For manifolds with boundary under a lower $N$-weighted Ricci curvature bound, and under a lower weighted mean curvature bound for the boundary, the author [33] has studied rigidity phenomena in the usual weighted case of $N \in[n, \infty]$. In the present paper, we produce rigidity theorems in the complementary weighted case of $N \in(-\infty, 1]$. Our rigidity theorems in the case of $N \in(-\infty, 1]$ give natural extensions of the corresponding results in [33].

To prove our rigidity theorems, we develop comparison theorems. We prove Laplacian comparison theorems for the distance function from the boundary, and volume comparison theorems for metric neighborhoods of the boundary. The author [33] has shown such comparison theorems in the usual weighted case of $N \in[n, \infty]$. For manifolds with boundary of non-negative $N$-weighted Ricci curvature, and of non-negative weighted mean curvature for the boundary, Wylie [39] has shown a Laplacian comparison inequality for the distance function from a connected component of the boundary in the weighted case of $N \in(-\infty, 1]$. To conclude our comparison theorems, we need slightly more complicated calculations than that done by the author [33], and by Wylie [39]. Under an assumption concerning a subharmonicity of the distance function from the boundary, we derive our rigidity theorems from studies of the equality cases in our comparison theorems.

1.1. Setting. We summarize our setting as follows: For $n \geq 2$, let $M$ be an $n$-dimensional, connected complete Riemannian manifold with boundary with Riemannian metric $g$. The boundary $\partial M$ is assumed to be smooth. We denote by $d_{M}$ the Riemannian distance on $M$ induced from the length structure determined by $g$. Let $f: M \rightarrow \mathbb{R}$ be a smooth function. For the Riemannian volume measure $\operatorname{vol}_{g}$ on $M$, let

$$
m_{f}:=e^{-f} \operatorname{vol}_{g} \text {. }
$$

For $N \in(-\infty, \infty]$, we denote by $\operatorname{Ric}_{f}^{N}$ the $N$-weighted Ricci curvature (see (1.1)). We note that for $N_{1}, N_{2} \in(-\infty, \infty] \backslash\{n\}$ with $N_{1} \leq N_{2}$, if $N_{1}, N_{2} \in(n, \infty]$ or $N_{1}, N_{2} \in(-\infty, n)$, then $\operatorname{Ric}_{f}^{N_{1}} \leq \operatorname{Ric}_{f}^{N_{2}}$; if $N_{1} \in$ 
$(-\infty, n)$ and $N_{2} \in(n, \infty]$, then $\operatorname{Ric}_{f}^{N_{2}} \leq \operatorname{Ric}_{f}^{N_{1}}$. We denote by $\operatorname{Ric}_{f, M}^{N}$ the infimum of $\operatorname{Ric}_{f}^{N}$ on the unit tangent bundle on the interior Int $M$ of $M$. For $x \in \partial M$, we denote by $u_{x}$ the unit inner normal vector on $\partial M$ at $x$. Let $H_{x}$ denote the mean curvature of $\partial M$ at $x$ defined as the trace of the shape operator of $u_{x}$. The $f$-mean curvature $H_{f, x}$ at $x$ is defined by

$$
H_{f, x}:=H_{x}+g\left((\nabla f)_{x}, u_{x}\right) .
$$

We put $H_{f, \partial M}:=\inf _{x \in \partial M} H_{f, x}$. Our main subject is a weighted Riemannian manifold $\left(M, d_{M}, m_{f}\right)$ with boundary such that for $\kappa, \lambda \in \mathbb{R}$ and for $N \in(-\infty, 1]$ we have $\operatorname{Ric}_{f, M}^{N} \geq \kappa$ and $H_{f, \partial M} \geq \lambda$.

1.2. Volume growth rigidity. Let $\rho_{\partial M}: M \rightarrow \mathbb{R}$ be the distance function from $\partial M$ defined as $\rho_{\partial M}(p):=d_{M}(p, \partial M)$. For $r \in(0, \infty)$, we put $B_{r}(\partial M):=\left\{p \in M \mid \rho_{\partial M}(p) \leq r\right\}$. For $x \in \partial M$, let $\gamma_{x}$ : $[0, T) \rightarrow M$ be the geodesic with initial conditions $\gamma_{x}(0)=x$ and $\gamma_{x}^{\prime}(0)=u_{x}$. We define a function $\tau: \partial M \rightarrow \mathbb{R} \cup\{\infty\}$ by

$$
\tau(x):=\sup \left\{t \in(0, \infty) \mid \rho_{\partial M}\left(\gamma_{x}(t)\right)=t\right\} .
$$

We define a function $F_{x}:[0, \tau(x)] \backslash\{\infty\} \rightarrow(0, \infty)$ by

$$
F_{x}(t):=e^{\frac{f\left(\gamma_{x}(t)\right)-f(x)}{n-1}} .
$$

Notice that if $f$ is constant, then $F_{x}$ is equal to 1 . For $\kappa, \lambda \in \mathbb{R}$, we say that $\kappa$ and $\lambda$ satisfy the subharmonic-condition if

$$
\inf _{x \in \partial M} \inf _{t \in(0, \tau(x))} \kappa \int_{0}^{t} F_{x}^{2}(s) d s \geq-\lambda .
$$

We remark that if $\kappa$ and $\lambda$ satisfy the subharmonic-condition, then subharmonicity of $\rho_{\partial M}$ is derived from $\operatorname{Ric}_{f, M}^{N} \geq \kappa$ and $H_{f, \partial M} \geq \lambda$ in the case of $N \in(-\infty, 1]$ (see Lemma 3.1). Note that if $\kappa, \lambda \in[0, \infty)$, then they satisfy the subharmonic-condition. We denote by $h$ the induced Riemnnian metric on $\partial M$. For the Riemannian volume measure $\operatorname{vol}_{h}$ on $\partial M$ induced from $h$, we put $m_{f, \partial M}:=e^{-\left.f\right|_{\partial M}} \operatorname{vol}_{h}$.

For an interval $I$, and for a Riemannian manifold $M_{0}$ with Riemannian metric $g_{0}$, let $\Phi: I \times M_{0} \rightarrow \mathbb{R}$ be a positive smooth function. For each $x \in M_{0}$, let $\Phi_{x}: I \rightarrow \mathbb{R}$ be the function defined as $\Phi_{x}(t):=\Phi(t, x)$. We say that a Riemannian manifold $\left(I \times M_{0}, d t^{2}+\Phi_{x}^{2}(t) g_{0}\right)$ is a twisted product space. When $\tau$ is infinity on $\partial M$, we define $[0, \infty) \times_{F} \partial M$ as the twisted product space $\left([0, \infty) \times \partial M, d t^{2}+F_{x}^{2}(t) h\right)$.

For the metric neighborhoods of the boundaries, we prove an absolute volume comparison theorem of Heintze-Karcher type, and a relative volume comparison theorem of Bishop-Gromov type (see Subsections 
4.1 and 4.2). We obtain rigidity results concerning the equality cases in those comparison theorems (see Subsection 4.3).

We conclude the following volume growth rigidity theorem:

Theorem 1.1. Let $M$ be a connected complete Riemannian manifold with boundary, and let $f: M \rightarrow \mathbb{R}$ be a smooth function. Suppose that $\partial M$ is compact. Let $\kappa \in \mathbb{R}$ and $\lambda \in \mathbb{R}$ satisfy the subharmoniccondition. For $N \in(-\infty, 1]$ we suppose $\operatorname{Ric}_{f, M}^{N} \geq \kappa$, and $H_{f, \partial M} \geq \lambda$. If we have

$$
\liminf _{r \rightarrow \infty} \frac{m_{f}\left(B_{r}(\partial M)\right)}{r} \geq m_{f, \partial M}(\partial M),
$$

then $\left(M, d_{M}\right)$ is isometric to $\left([0, \infty) \times_{F} \partial M, d_{[0, \infty) \times_{F} \partial M}\right)$. Moreover, if $N \in(-\infty, 1)$, then for every $x \in \partial M$ the function $f \circ \gamma_{x}$ is constant on $[0, \infty)$; in particular, $\left(M, d_{M}\right)$ is isometric to $\left([0, \infty) \times \partial M, d_{[0, \infty) \times \partial M}\right)$.

When $\kappa=0$ and $\lambda=0$, Theorem 1.1 has been proved in the unweighted case in [32], and in the usual weighted case in [33].

Remark 1.1. We do not know whether Theorem 1.1 can be extended to the weighted case of $N \in(1, n)$.

Remark 1.2. Under the same setting as in Theorem 1.1, we always have the following inequality (see Lemma 4.1):

$$
\limsup _{r \rightarrow \infty} \frac{m_{f}\left(B_{r}(\partial M)\right)}{r} \leq m_{f, \partial M}(\partial M) .
$$

Theorem 1.1 is concerned with rigidity phenomena.

We have the following corollary of Theorem 1.1 .

Corollary 1.2. Under the same setting as in Theorem 1.1, if $N=$ 1 and $\kappa=0$, and if we have (1.6), then there exist a function $f_{0}$ : $[0, \infty) \rightarrow \mathbb{R}$, and a Riemannian metric $h_{0}$ on $\partial M$ such that $M$ is isometric to a warped product space $\left([0, \infty) \times \partial M, d t^{2}+e^{2 \frac{f_{0}(t)}{n-1}} h_{0}\right)$.

1.3. Splitting theorems. In our setting, we show Laplacian comparison theorems for $\rho_{\partial M}$, and study the equality cases (see Section 3).

By using a Laplacian comparison theorem for $\rho_{\partial M}$, and that for Busemann functions, we prove the following splitting theorem:

Theorem 1.3. Let $M$ be a connected complete Riemannian manifold with boundary, and let $f: M \rightarrow \mathbb{R}$ be a smooth function such that $\sup f(M)<\infty$. For $N \in(-\infty, 1]$ we suppose $\operatorname{Ric}_{f, M}^{N} \geq 0$, and $H_{f, \partial M} \geq$ 0 . If for some $x_{0} \in \partial M$ we have $\tau\left(x_{0}\right)=\infty$, then $\left(M, d_{M}\right)$ is isometric to $\left([0, \infty) \times_{F} \partial M, d_{[0, \infty) \times_{F} \partial M}\right)$. Moreover, if $N \in(-\infty, 1)$, then for 
every $x \in \partial M$ the function $f \circ \gamma_{x}$ is constant on $[0, \infty)$; in particular, $\left(M, d_{M}\right)$ is isometric to $\left([0, \infty) \times \partial M, d_{[0, \infty) \times \partial M}\right)$.

In the unweighted case, Kasue [13] has proved Theorem 1.3 under the compactness assumption for the boundary (see also the work of Croke and Kleiner [9]). Theorem 1.3 itself has been proved in the unweighted case in [32, and in the usual weighted case in [33].

Remark 1.3. We do not know whether Theorem 1.3 can be extended to the weighted case of $N \in(1, n)$.

As a corollary of Theorem 1.3 , we see the following:

Corollary 1.4. Under the same setting as in Theorem 1.3, if $N=1$, and if for some $x_{0} \in \partial M$ we have $\tau\left(x_{0}\right)=\infty$, then there exist a function $f_{0}:[0, \infty) \rightarrow \mathbb{R}$, and a Riemannian metric $h_{0}$ on $\partial M$ such that $M$ is isometric to $\left([0, \infty) \times \partial M, d t^{2}+e^{2 \frac{f_{0}(t)}{n-1}} h_{0}\right)$.

In Theorem 1.3. by applying the Wylie splitting theorem in 39 to the boundary, we obtain a multi-splitting theorem (see Subsection 5.3). We also generalize a splitting theorem studied in [13] (and [9], [12]) for the case where boundaries are disconnected (see Subsection 5.4).

1.4. Eigenvalue rigidity. For $p \in[1, \infty)$, the $(1, p)$-Sobolev space $W_{0}^{1, p}\left(M, m_{f}\right)$ on $\left(M, m_{f}\right)$ with compact support is defined as the completion of the set of all smooth functions on $M$ whose support is compact and contained in Int $M$ with respect to the standard $(1, p)$-Sobolev norm. We denote by $\|\cdot\|$ the standard norm induced from $g$, and by div the divergence with respect to $g$. For $p \in[1, \infty)$, the $(f, p)$-Laplacian $\Delta_{f, p} \varphi$ for $\varphi \in W_{0}^{1, p}\left(M, m_{f}\right)$ is defined by

$$
\Delta_{f, p} \varphi:=-e^{f} \operatorname{div}\left(e^{-f}\|\nabla \varphi\|^{p-2} \nabla \varphi\right)
$$

as a distribution on $W_{0}^{1, p}\left(M, m_{f}\right)$. A real number $\mu$ is said to be an $(f, p)$-Dirichlet eigenvalue for $\Delta_{f, p}$ on $M$ if there exists $\varphi \in W_{0}^{1, p}\left(M, m_{f}\right) \backslash$ $\{0\}$ such that $\Delta_{f, p} \varphi=\mu|\varphi|^{p-2} \varphi$ holds on Int $M$ in a distribution sense on $W_{0}^{1, p}\left(M, m_{f}\right)$. For $p \in[1, \infty)$, the Rayleigh quotient $R_{f, p}(\varphi)$ for $\varphi \in W_{0}^{1, p}\left(M, m_{f}\right) \backslash\{0\}$ is defined as

$$
R_{f, p}(\varphi):=\frac{\int_{M}\|\nabla \varphi\|^{p} d m_{f}}{\int_{M}|\varphi|^{p} d m_{f}} .
$$

We put $\mu_{f, 1, p}(M):=\inf _{\varphi} R_{f, p}(\varphi)$, where the infimum is taken over all non-zero functions in $W_{0}^{1, p}\left(M, m_{f}\right)$. The value $\mu_{f, 1,2}(M)$ is equal to the infimum of the spectrum of $\Delta_{f, 2}$ on $\left(M, m_{f}\right)$. If $M$ is compact, and 
if $p \in(1, \infty)$, then $\mu_{f, 1, p}(M)$ is equal to the infimum of the set of all $(f, p)$-Dirichlet eigenvalues on $M$.

Let $p \in(1, \infty)$. For $D \in(0, \infty)$, let $\mu_{p, D}$ be the positive minimum real number $\mu$ such that there exists a function $\varphi:[0, D] \rightarrow \mathbb{R}$ satisfying

$$
\left(\left|\varphi^{\prime}(t)\right|^{p-2} \varphi^{\prime}(t)\right)^{\prime}+\mu|\varphi(t)|^{p-2} \varphi(t)=0, \quad \varphi(0)=0, \quad \varphi^{\prime}(D)=0 .
$$

In the case where $p=2$, we see $\mu_{2, D}=\pi^{2}(2 D)^{-2}$.

For a positive number $D \in(0, \infty)$, and for a connected component $\partial M_{1}$ of $\partial M$, we denote by $[0, D] \times_{F} \partial M_{1}$ the twisted product space $\left([0, D] \times \partial M_{1}, d t^{2}+F_{x}^{2}(t) h\right)$, where for every $x \in \partial M_{1}$ the function $F_{x}:[0, D] \rightarrow(0, \infty)$ is defined as $(1.5)$. The inscribed radius of $M$ is defined as

$$
D(M, \partial M):=\sup _{p \in M} \rho_{\partial M}(p) .
$$

Suppose that $M$ is compact. We say that the metric space $\left(M, d_{M}\right)$ is an $F$-model space if $M$ is isometric to either (1) for a connected component $\partial M_{1}$ of $\partial M$, the twisted product space $[0,2 D(M, \partial M)] \times_{F} \partial M_{1}$; or (2) for an involutive isometry $\sigma$ of $\partial M$ without fixed points, the quotient space $\left([0,2 D(M, \partial M)] \times_{F} \partial M\right) / G_{\sigma}$, where $G_{\sigma}$ is the isometry group on $[0,2 D(M, \partial M)] \times_{F} \partial M$ of the identity and the involute isometry $\hat{\sigma}$ defined by $\hat{\sigma}(t, x):=(2 D(M, \partial M)-t, \sigma(x))$. If $\left(M, d_{M}\right)$ is an $F$ model space, and if for every $x \in \partial M$ the function $F_{x}$ is equal to 1 on $[0, D(M, \partial M)]$, then we call the $F$-model space $\left(M, d_{M}\right)$ an equational model space. The notion of the equational model spaces coincides with that of the $(0,0)$-equational model spaces introduced in [33].

We prove the following rigidity theorem for $\mu_{f, 1, p}$ :

Theorem 1.5. Let $M$ be a connected complete Riemannian manifold with boundary, and let $f: M \rightarrow \mathbb{R}$ be a smooth function. Suppose that $M$ is compact. Let $p \in(1, \infty)$, and let $\kappa \in \mathbb{R}$ and $\lambda \in \mathbb{R}$ satisfy the subharmonic-condition. For $N \in(-\infty, 1]$ we suppose $\operatorname{Ric}_{f, M}^{N} \geq \kappa$, and $H_{f, \partial M} \geq \lambda$. For $D \in(0, \infty)$ we assume $D(M, \partial M) \leq D$. Then

$$
\mu_{f, 1, p}(M) \geq \mu_{p, D}
$$

If the equality in (1.9) holds, then $D(M, \partial M)=D$, and the metric space $\left(M, d_{M}\right)$ is an F-model space. Moreover, if $N \in(-\infty, 1)$, then for every $x \in \partial M$ the function $f \circ \gamma_{x}$ is constant on $[0, D]$; in particular, $\left(M, d_{M}\right)$ is an equational model space.

In the unweighted case, Li and Yau [18] have obtained the estimate (1.9), and Kasue [14] has proved Theorem 1.5 when $p=2, \kappa=0$ and $\lambda=0$. In [33], the author has proved Theorem 1.3 in the usual weighted case when $\kappa=0$ and $\lambda=0$. 
Remark 1.4. We do not know whether Theorem 1.5 can be extended to the weighted case of $N \in(1, n)$.

Suppose that $M$ is compact. We say that the metric space $\left(M, d_{M}\right)$ is a warped model space if there exist a function $f_{0}:[0,2 D(M, \partial M)] \rightarrow \mathbb{R}$, and a Riemannian metric $h_{0}$ on $\partial M$ such that $M$ is isometric to either (1) for a connected component $\partial M_{1}$ of $\partial M$, the warped product space $\left([0,2 D(M, \partial M)] \times \partial M_{1}, d t^{2}+e^{2 \frac{f_{0}(t)}{n-1}} h_{0}\right)$; or (2) for an involutive isometry $\sigma$ of $\partial M$ without fixed points, the quotient space $\left([0,2 D(M, \partial M)] \times \partial M, d t^{2}+e^{2 \frac{f_{0}(t)}{n-1}} h_{0}\right) / G_{\sigma}$, where $G_{\sigma}$ is the isometry group on $\left([0,2 D(M, \partial M)] \times \partial M, d t^{2}+e^{2 \frac{f_{0}(t)}{n-1}} h_{0}\right)$ of the identity and the involute isometry $\hat{\sigma}$ defined as $\hat{\sigma}(t, x):=(2 D(M, \partial M)-t, \sigma(x))$.

We obtain the following corollary of Theorem 1.5 .

Corollary 1.6. Under the same setting as in Theorem 1.5, if $N=1$ and $\kappa=0$, and if the equality in (1.9) holds, then the metric space $\left(M, d_{M}\right)$ is a warped model space.

1.5. Organization. In Section 2, we prepare some notations and recall the basic facts for Riemannian manifolds with boundary. In Section 3 , we show Laplacian comparison results for $\rho_{\partial M}$. In Section 4, we show volume comparison results, and conclude Theorem 1.1 and Corollary 1.2. In Section 5, we prove Theorem 1.3 and Corollary 1.4, and discuss its variants. In Section 6, we prove Theorem 1.5 and Corollary 1.6. We also obtain an explicit lower bound for $\mu_{f, 1, p}$ (see Subsection 6.3).

Acknowledgements. The author would like to express his gratitude to Professor Koichi Nagano for his constant advice and suggestions. The author would also like to thank Professor Shin-ichi Ohta for his valuable comments. The author would like to thank Professor William Wylie for his valuable advice concerning Proposition 2.9.

\section{Preliminaries}

We refer to [31] for the basics of Riemannian manifolds with boundary (cf. Section 2 in [32], and in [33]).

2.1. Riemannian manifolds with boundary. For $n \geq 2$, let $M$ be an $n$-dimensional, connected Riemannian manifold with (smooth) boundary with Riemannian metric $g$. For a point $p \in \operatorname{Int} M$, let $T_{p} M$ be the tangent space at $p$ on $M$, and let $U_{p} M$ be the unit tangent sphere at $p$ on $M$. We denote by $\|\cdot\|$ the standard norm induced from $g$. If $v_{1}, \ldots, v_{k} \in T_{p} M$ are linearly independent, then it holds that $\left\|v_{1} \wedge \cdots \wedge v_{k}\right\|=\sqrt{\operatorname{det}\left(g\left(v_{i}, v_{j}\right)\right)}$. 
Let $d_{M}$ be the Riemannian distance on $M$ induced from the length structure determined by $g$. For an interval $I$, we say that a curve $\gamma: I \rightarrow M$ is a normal minimal geodesic if for all $s, t \in I$ we have $d_{M}(\gamma(s), \gamma(t))=|s-t|$, and $\gamma$ is a normal geodesic if for each $t \in I$ there exists an interval $J \subset I$ with $t \in J$ such that $\left.\gamma\right|_{J}$ is a normal minimal geodesic. If $M$ is complete with respect to $d_{M}$, then the HopfRinow theorem for length spaces (see e.g., Theorem 2.5.23 in [5]) tells us that the metric space $\left(M, d_{M}\right)$ is a proper, geodesic space; namely, all closed bounded subsets of $M$ are compact, and for every pair of points in $M$ there exists a normal minimal geodesic connecting them.

For $i=1,2$, let $M_{i}$ be connected Riemannian manifolds with boundary with Riemannian metric $g_{i}$. For each $i$, the boundary $\partial M_{i}$ carries the induced Riemannian metric $h_{i}$. We say that a homeomorphism $\Phi: M_{1} \rightarrow M_{2}$ is a Riemannian isometry with boundary from $M_{1}$ to $M_{2}$ if $\Phi$ satisfies the following conditions:

(1) $\left.\Phi\right|_{\operatorname{Int} M_{1}}: \operatorname{Int} M_{1} \rightarrow \operatorname{Int} M_{2}$ is smooth, and $\left(\left.\Phi\right|_{\operatorname{Int} M_{1}}\right)^{*}\left(g_{2}\right)=g_{1}$;

(2) $\left.\Phi\right|_{\partial M_{1}}: \partial M_{1} \rightarrow \partial M_{2}$ is smooth, and $\left(\left.\Phi\right|_{\partial M_{1}}\right)^{*}\left(h_{2}\right)=h_{1}$.

If $\Phi: M_{1} \rightarrow M_{2}$ is a Riemannian isometry with boundary, then the inverse $\Phi^{-1}$ is also a Riemannian isometry with boundary. Notice that there exists a Riemannian isometry with boundary from $M_{1}$ to $M_{2}$ if and only if the metric space $\left(M_{1}, d_{M_{1}}\right)$ is isometric to $\left(M_{2}, d_{M_{2}}\right)$ (see e.g., Section 2 in [32]).

2.2. Jacobi fields orthogonal to the boundary. Let $M$ be a connected Riemannian manifold with boundary with Riemannian metric $g$. For a point $x \in \partial M$, and for the tangent space $T_{x} \partial M$ at $x$ on $\partial M$, let $T_{x}^{\perp} \partial M$ be the orthogonal complement of $T_{x} \partial M$ in the tangent space at $x$ on $M$. Take $u \in T_{x}^{\perp} \partial M$. For the second fundamental form $S$ of $\partial M$, let $A_{u}: T_{x} \partial M \rightarrow T_{x} \partial M$ be the shape operator for $u$ defined as

$$
g\left(A_{u} v, w\right):=g(S(v, w), u) .
$$

We denote by $u_{x}$ the unit inner normal vector at $x$. The mean curvature $H_{x}$ at $x$ is defined as $H_{x}:=\operatorname{trace} A_{u_{x}}$. We denote by $\gamma_{x}:[0, T) \rightarrow M$ the normal geodesic with initial conditions $\gamma_{x}(0)=x$ and $\gamma_{x}^{\prime}(0)=u_{x}$. We say that a Jacobi field $Y$ along $\gamma_{x}$ is a $\partial M$-Jacobi field if $Y$ satisfies the following initial conditions:

$$
Y(0) \in T_{x} \partial M, \quad Y^{\prime}(0)+A_{u_{x}} Y(0) \in T_{x}^{\perp} \partial M .
$$

We say that $\gamma_{x}\left(t_{0}\right)$ is a conjugate point of $\partial M$ along $\gamma_{x}$ if there exists a non-zero $\partial M$-Jacobi field $Y$ along $\gamma_{x}$ with $Y\left(t_{0}\right)=0$. We denote by $\tau_{1}(x)$ the first conjugate value for $\partial M$ along $\gamma_{x}$. It is well-known that for all $x \in \partial M$ and $t>\tau_{1}(x)$, we have $t>\rho_{\partial M}\left(\gamma_{x}(t)\right)$. 
For the normal tangent bundle $T^{\perp} \partial M:=\bigcup_{x \in \partial M} T_{x}^{\perp} \partial M$ of $\partial M$, let $0\left(T^{\perp} \partial M\right)$ be the zero-section $\bigcup_{x \in \partial M}\left\{0_{x} \in T_{x}^{\perp} \partial M\right\}$ of $T^{\perp} \partial M$. On an open neighborhood of $0\left(T^{\perp} \partial M\right)$ in $T^{\perp} \partial M$, the normal exponential map $\exp ^{\perp}$ of $\partial M$ is defined as $\exp ^{\perp}(x, u):=\gamma_{x}(\|u\|)$ for $x \in \partial M$ and $u \in T_{x}^{\perp} \partial M$.

For $x \in \partial M$ and $t \in\left[0, \tau_{1}(x)\right)$, we denote by $\theta(t, x)$ the absolute value of the Jacobian of $\exp ^{\perp}$ at $\left(x, t u_{x}\right) \in T^{\perp} \partial M$. For each $x \in \partial M$, we choose an orthonomal basis $\left\{e_{x, i}\right\}_{i=1}^{n-1}$ of $T_{x} \partial M$. For each $i$, let $Y_{x, i}$ be the $\partial M$-Jacobi field along $\gamma_{x}$ with initial conditions $Y_{x, i}(0)=e_{x, i}$ and $Y_{x, i}^{\prime}(0)=-A_{u_{x}} e_{x, i}$. Note that for all $x \in \partial M$ and $t \in\left[0, \tau_{1}(x)\right)$, we have $\theta(t, x)=\left\|Y_{x, 1}(t) \wedge \cdots \wedge Y_{x, n-1}(t)\right\|$. This does not depend on the choice of the orthonormal bases.

2.3. Cut locus for the boundary. We recall the basic properties of the cut locus for the boundary. The basic properties seem to be well-known. We refer to 32 for the proofs.

Let $M$ be a connected complete Riemannian manifold with boundary with Riemannian metric $g$. For $p \in M$, we call $x \in \partial M$ a foot point on $\partial M$ of $p$ if $d_{M}(p, x)=\rho_{\partial M}(p)$. Since $\left(M, d_{M}\right)$ is proper, every point in $M$ has at least one foot point on $\partial M$. For $p \in \operatorname{Int} M$, let $x \in \partial M$ be a foot point on $\partial M$ of $p$. Then there exists a unique normal minimal geodesic $\gamma:[0, l] \rightarrow M$ from $x$ to $p$ such that $\gamma=\left.\gamma_{x}\right|_{[0, l]}$, where $l=\rho_{\partial M}(p)$. In particular, $\gamma^{\prime}(0)=u_{x}$ and $\left.\gamma\right|_{(0, l]}$ lies in Int $M$.

Let $\tau: \partial M \rightarrow \mathbb{R} \cup\{\infty\}$ be the function defined as (1.4). By the property of $\tau_{1}$, for all $x \in \partial M$ we have $0<\tau(x) \leq \tau_{1}(x)$. For the inscribed radius $D(M, \partial M)$ of $M$, from the definition of $\tau$, we have $D(M, \partial M)=\sup _{x \in \partial M} \tau(x)$. The function $\tau$ is continuous on $\partial M$.

The continuity of $\tau$ implies the following (see e.g., Section 3 in [32]):

Lemma 2.1. Suppose that $\partial M$ is compact. Then $D(M, \partial M)$ is finite if and only if $M$ is compact.

We put

$$
\begin{aligned}
T D_{\partial M} & :=\bigcup_{x \in \partial M}\left\{t u_{x} \in T_{x}^{\perp} \partial M \mid t \in[0, \tau(x))\right\}, \\
T \text { Cut } \partial M & :=\bigcup_{x \in \partial M}\left\{\tau(x) u_{x} \in T_{x}^{\perp} \partial M \mid \tau(x)<\infty\right\},
\end{aligned}
$$

and define $D_{\partial M}:=\exp ^{\perp}\left(T D_{\partial M}\right)$ and Cut $\partial M:=\exp ^{\perp}(T$ Cut $\partial M)$. We call Cut $\partial M$ the cut locus for the boundary $\partial M$. From the continuity of $\tau$, the set Cut $\partial M$ is a null set of $M$. Furthermore, we have

$$
\text { Int } M=\left(D_{\partial M} \backslash \partial M\right) \sqcup \operatorname{Cut} \partial M, \quad M=D_{\partial M} \sqcup \operatorname{Cut} \partial M \text {. }
$$


This implies that if Cut $\partial M=\emptyset$, then $\partial M$ is connected. The set $T D_{\partial M} \backslash 0\left(T^{\perp} \partial M\right)$ is a maximal domain in $T^{\perp} \partial M$ on which $\exp ^{\perp}$ is regular and injective.

In [33], we have already known the following:

Lemma 2.2. If there exists a connected component $\partial M_{0}$ of $\partial M$ such that for all $x \in \partial M_{0}$ we have $\tau(x)=\infty$, then $\partial M$ is connected and Cut $\partial M=\emptyset$.

The function $\rho_{\partial M}$ is smooth on Int $M \backslash$ Cut $\partial M$. For each $p \in$ Int $M \backslash$ Cut $\partial M$, the gradient vector $\nabla \rho_{\partial M}(p)$ of $\rho_{\partial M}$ at $p$ is given by $\nabla \rho_{\partial M}(p)=\gamma^{\prime}(l)$, where $\gamma:[0, l] \rightarrow M$ is the normal minimal geodesic from the foot point on $\partial M$ of $p$ to $p$.

For $\Omega \subset M$, we denote by $\bar{\Omega}$ the closure of $\Omega$ in $M$, and by $\partial \Omega$ the boundary of $\Omega$ in $M$. For a domain $\Omega$ in $M$ such that $\partial \Omega$ is a smooth hypersurface in $M$, we denote by $\operatorname{vol}_{\partial \Omega}$ the canonical Riemannian volume measure on $\partial \Omega$.

We have the following fact to avoid the cut locus for the boundary that has been stated in [33] (see Lemma 2.6 in [33]):

Lemma 2.3. Let $\Omega$ be a domain in $M$ such that $\partial \Omega$ is a smooth hypersurface in $M$. Then there exists a sequence $\left\{\Omega_{k}\right\}_{k \in \mathbb{N}}$ of closed subsets of $\bar{\Omega}$ such that for every $k \in \mathbb{N}$, the set $\partial \Omega_{k}$ is a smooth hypersurface in $M$ except for a null set in $\left(\partial \Omega, \operatorname{vol}_{\partial \Omega}\right)$ satisfying the following properties:

(1) for all $k_{1}, k_{2} \in \mathbb{N}$ with $k_{1}<k_{2}$, we have $\Omega_{k_{1}} \subset \Omega_{k_{2}}$;

(2) $\bar{\Omega} \backslash \operatorname{Cut} \partial M=\bigcup_{k \in \mathbb{N}} \Omega_{k}$;

(3) for every $k \in \mathbb{N}$, and for almost every point $p \in \partial \Omega_{k} \cap \partial \Omega$ in $\left(\partial \Omega, \operatorname{vol}_{\partial \Omega}\right)$, there exists the unit outer normal vector for $\Omega_{k}$ at $p$ that coincides with the unit outer normal vector on $\partial \Omega$ for $\Omega$ at $p$;

(4) for every $k \in \mathbb{N}$, on $\partial \Omega_{k} \backslash \partial \Omega$, there exists the unit outer normal vector field $\nu_{k}$ for $\Omega_{k}$ such that $g\left(\nu_{k}, \nabla \rho_{\partial M}\right) \geq 0$.

Moreover, if $\bar{\Omega}=M$, then for every $k \in \mathbb{N}$, the set $\partial \Omega_{k}$ is a smooth hypersurface in $M$, and satisfies $\partial \Omega_{k} \cap \partial M=\partial M$.

As noticed in [33], for the cut locus for a single point, we have known a similar fact to Lemma 2.3 (see e.g., Theorem 4.1 in [7]). One can prove Lemma 2.3 by a similar method to the case of the cut locus for a single point.

2.4. Busemann functions and asymptotes. Let $M$ be a connected complete Riemannian manifold with boundary. A normal geodesic $\gamma$ : $[0, \infty) \rightarrow M$ is said to be a ray if for all $s, t \in[0, \infty)$ it holds that 
$d_{M}(\gamma(s), \gamma(t))=|s-t|$. For a ray $\gamma:[0, \infty) \rightarrow M$, the Busemann function $b_{\gamma}: M \rightarrow \mathbb{R}$ of $\gamma$ is defined as

$$
b_{\gamma}(p):=\lim _{t \rightarrow \infty}\left(t-d_{M}(p, \gamma(t))\right) \text {. }
$$

Take a ray $\gamma:[0, \infty) \rightarrow M$ and a point $p \in$ Int $M$, and choose a sequence $\left\{t_{i}\right\}$ with $t_{i} \rightarrow \infty$. For each $i$, we take a normal minimal geodesic $\gamma_{i}:\left[0, l_{i}\right] \rightarrow M$ from $p$ to $\gamma\left(t_{i}\right)$. Since $\gamma$ is a ray, it follows that $l_{i} \rightarrow \infty$. Take a sequence $\left\{T_{j}\right\}$ with $T_{j} \rightarrow \infty$. Using the fact that $M$ is proper, we take a subsequence $\left\{\gamma_{1, i}\right\}$ of $\left\{\gamma_{i}\right\}$, and a normal minimal geodesic $\gamma_{p, 1}:\left[0, T_{1}\right] \rightarrow M$ from $p$ to $\gamma_{p, 1}\left(T_{1}\right)$ such that $\left.\gamma_{1, i}\right|_{\left[0, T_{1}\right]}$ uniformly converges to $\gamma_{p, 1}$. In this manner, take a subsequence $\left\{\gamma_{2, i}\right\}$ of $\left\{\gamma_{1, i}\right\}$ and a normal minimal geodesic $\gamma_{p, 2}:\left[0, T_{2}\right] \rightarrow M$ from $p$ to $\gamma_{p, 2}\left(T_{2}\right)$ such that $\left.\gamma_{2, i}\right|_{\left[0, T_{2}\right]}$ uniformly converges to $\gamma_{p, 2}$, where $\left.\gamma_{p, 2}\right|_{\left[0, T_{1}\right]}=\gamma_{p, 1}$. By means of a diagonal argument, we obtain a subsequence $\left\{\gamma_{k}\right\}$ of $\left\{\gamma_{i}\right\}$ and a ray $\gamma_{p}$ in $M$ such that for every $t \in(0, \infty)$ we have $\gamma_{k}(t) \rightarrow \gamma_{p}(t)$ as $k \rightarrow \infty$. We call such a ray $\gamma_{p}$ an asymptote for $\gamma$ from $p$.

The following lemmas have been shown in [32].

Lemma 2.4. Suppose that for some $x \in \partial M$ we have $\tau(x)=\infty$. Take $p \in \operatorname{Int} M$. If $b_{\gamma_{x}}(p)=\rho_{\partial M}(p)$, then $p \notin$ Cut $\partial M$. Moreover, for the unique foot point $y$ on $\partial M$ of $p$, we have $\tau(y)=\infty$.

Lemma 2.5. Suppose that for some $x \in \partial M$ we have $\tau(x)=\infty$. For $l \in(0, \infty)$, put $p:=\gamma_{x}(l)$. Then there exists $\epsilon \in(0, \infty)$ such that for all $q \in B_{\epsilon}(p)$, all asymptotes for the ray $\gamma_{x}$ from $q$ lie in $\operatorname{Int} M$.

2.5. Weighted Riemannian manifolds with boundary. Let $M$ be a connected complete Riemannian manifold with boundary, and let $f: M \rightarrow \mathbb{R}$ be a smooth function. For a smooth function $\varphi$ on $M$, the weighted Laplacian $\Delta_{f} \varphi$ for $\varphi$ is defined by

$$
\Delta_{f} \varphi:=\Delta \varphi+g(\nabla f, \nabla \varphi)
$$

where $\Delta \varphi$ is the Laplacian for $\varphi$ defined as the minus of the trace of its Hessian. Note that $\Delta_{f}$ coincides with the $(f, 2)$-Laplacian $\Delta_{f, 2}$.

It seems that the following formula of Bochner type is well-known (see [19], and Chapter 14 in [36]).

Proposition 2.6 ([19]). For every smooth function $\varphi$ on $M$, we have

$$
-\frac{1}{2} \Delta_{f}\|\nabla \varphi\|^{2}=\operatorname{Ric}_{f}^{\infty}(\nabla \varphi)+\|\operatorname{Hess} \varphi\|^{2}-g\left(\nabla \Delta_{f} \varphi, \nabla \varphi\right),
$$

where $\|$ Hess $\varphi \|$ is the Hilbert-Schmidt norm of Hess $\varphi$.

Notice that for every $x \in \partial M$, and for every $t \in(0, \tau(x))$, the value $\Delta \rho_{\partial M}\left(\gamma_{x}(t)\right)$ is equal to the mean curvature $H_{x, t}$ of the $t$-level set of 
$\rho_{\partial M}$ at $\gamma_{x}(t)$ toward $\nabla \rho_{\partial M}$. In our weighted case, by the definition of the weighted Laplacian, we see the following:

Lemma 2.7. Take $x \in \partial M$. Then for every $t \in(0, \tau(x))$, the value $\Delta_{f} \rho_{\partial M}\left(\gamma_{x}(t)\right)$ is equal to the $f$-mean curvature $H_{f, x, t}$ of the t-level set of $\rho_{\partial M}$ at $\gamma_{x}(t)$ toward $\nabla \rho_{\partial M}$ defined as

$$
H_{f, x, t}:=H_{x, t}+g\left(\nabla f, \nabla \rho_{\partial M}\right)\left(\gamma_{x}(t)\right) .
$$

In particular, $\Delta_{f} \rho_{\partial M}\left(\gamma_{x}(t)\right)$ tends to $H_{f, x}$ as $t \rightarrow 0$, where $H_{f, x}$ is the $f$-mean curvature of $\partial M$ at $x$ defined as $(1.3)$.

For $x \in \partial M$ and $t \in[0, \tau(x))$, we put

$$
\theta_{f}(t, x):=e^{-f\left(\gamma_{x}(t)\right)} \theta(t, x),
$$

where $\theta(t, x)$ is the absolute value of the Jacobian of the map $\exp ^{\perp}$ at $\left(x, t u_{x}\right) \in T^{\perp} \partial M$. For all $x \in \partial M$ and $t \in(0, \tau(x))$, it holds that

$$
\Delta_{f} \rho_{\partial M}\left(\gamma_{x}(t)\right)=-(\log \theta(t, x))^{\prime}+f\left(\gamma_{x}(t)\right)^{\prime}=-\frac{\theta_{f}^{\prime}(t, x)}{\theta_{f}(t, x)} .
$$

Let $\bar{\theta}_{f}:[0, \infty) \times \partial M \rightarrow \mathbb{R}$ be a function defined by

$$
\bar{\theta}_{f}(t, x):= \begin{cases}\theta_{f}(t, x) & \text { if } t<\tau(x) \\ 0 & \text { if } t \geq \tau(x) .\end{cases}
$$

The following has been shown in [33]:

Lemma 2.8. If $\partial M$ is compact, then for all $r \in(0, \infty)$

$$
m_{f}\left(B_{r}(\partial M)\right)=\int_{\partial M} \int_{0}^{r} \bar{\theta}_{f}(t, x) d t d \operatorname{vol}_{h}
$$

where $m_{f}$ denotes the weighted measure on $M$ defined as (1.2), and $h$ denotes the induced Riemannian metric on $\partial M$.

2.6. Twisted and warped product spaces. In [39], for the proof of a splitting theorem of Cheeger-Gromoll type, Wylie has proved that a twisted product space over $\mathbb{R}$ becomes a warped product space under a non-negativity of the 1-weighted Ricci curvature (see Proposition 2.2 in [39]). The proof is based on a pointwise calculation, and the same holds true for a twisted product space over an arbitrary interval.

From the argument in the proof of Proposition 2.2 in [39], we can derive the following in our setting:

Proposition 2.9 ([39]). Let $M$ be a Riemannian manifold with boundary, and let $f: M \rightarrow \mathbb{R}$ be a smooth function. Suppose that there exist an interval $I$ in the form of $[0, \infty)$ or $[0, D]$ for some positive number $D$, and a connected component $\partial M_{1}$ of $\partial M$ such that $M$ is isometric 
to a twisted product space $I \times_{F} \partial M_{1}$. If $\operatorname{Ric}_{f, M}^{1} \geq 0$, then there exist functions $f_{0}: I \rightarrow \mathbb{R}$ and $f_{1}: \partial M_{1} \rightarrow \mathbb{R}$ such that for all $t \in I$ and $x \in \partial M_{1}$ we have $f\left(\gamma_{x}(t)\right)=f_{0}(t)+f_{1}(x)$; in particular, $M$ is isometric to a warped product space $\left(I \times \partial M_{1}, d t^{2}+e^{\frac{2 f_{0}(t)}{n-1}} h_{0}\right)$, where for the induced metric $h$ on $\partial M_{1}$ we put $h_{0}:=e^{2 \frac{f_{1}-\left(\left.f\right|_{\left.\partial M_{1}\right)}\right.}{n-1}} h$.

Notice that Proposition 2.9 has been implicitly used in the proof of Theorem 5.1 in 39 .

\section{LAPLACIAN COMPARISONS}

In this section, let $M$ be an $n$-dimensional, connected complete Riemannian manifold with boundary with Riemannian metric $g$, and let $f: M \rightarrow \mathbb{R}$ be a smooth function.

3.1. Basic comparisons. Recall that for $x \in \partial M$, the function $F_{x}$ : $[0, \tau(x)] \backslash\{\infty\} \rightarrow(0, \infty)$ is defined as 1.5$)$. For $\kappa, \lambda \in \mathbb{R}$, we define a function $F_{\kappa, \lambda, x}:[0, \tau(x)] \backslash\{\infty\} \rightarrow \mathbb{R}$ by

$$
F_{\kappa, \lambda, x}(t):=\kappa \int_{0}^{t} F_{x}^{2}(s) d s+\lambda .
$$

Note that if $\kappa$ and $\lambda$ satisfy the subharmonic-condition, then for every $x \in \partial M$ the function $F_{\kappa, \lambda, x}$ is non-negative.

We have the following Laplacian comparison inequality for $\rho_{\partial M}$ :

Lemma 3.1. Take $x \in \partial M$. For $\kappa, \lambda \in \mathbb{R}$ and for $N \in(-\infty, 1]$ we suppose that for all $t \in(0, \tau(x))$ we have $\operatorname{Ric}_{f}^{N}\left(\gamma_{x}^{\prime}(t)\right) \geq \kappa$, and suppose $H_{f, x} \geq \lambda$. Then for all $t \in(0, \tau(x))$ we have

$$
\Delta_{f} \rho_{\partial M}\left(\gamma_{x}(t)\right) \geq F_{x}^{-2}(t) F_{\kappa, \lambda, x}(t) .
$$

In particular, if $\kappa$ and $\lambda$ satisfy the subharmonic-condition, then for all $t \in(0, \tau(x))$ we have $\Delta_{f} \rho_{\partial M}\left(\gamma_{x}(t)\right) \geq 0$.

Proof. The function $\rho_{\partial M} \circ \gamma_{x}$ is smooth on $(0, \tau(x))$. We put $h_{f, x}:=$ $\left(\Delta_{f} \rho_{\partial M}\right) \circ \gamma_{x}$. We first show that for all $s \in(0, \tau(x))$

$$
\left(F_{x}^{2}(s) h_{f, x}(s)-\kappa \int_{0}^{s} F_{x}^{2}(u) d u\right)^{\prime} \geq 0 .
$$

Fix $s \in(0, \tau(x))$, and put $f_{x}:=f \circ \gamma_{x}$. We apply Proposition 2.6 to the function $\rho_{\partial M}$. Since $\left\|\nabla \rho_{\partial M}\right\|=1$ along $\left.\gamma_{x}\right|_{(0, \tau(x))}$, it holds that

$$
\begin{aligned}
0 & =\operatorname{Ric}_{f}^{\infty}\left(\gamma_{x}^{\prime}(s)\right)+\left\|\operatorname{Hess} \rho_{\partial M}\right\|^{2}\left(\gamma_{x}(s)\right)-g\left(\nabla \Delta_{f} \rho_{\partial M}, \nabla \rho_{\partial M}\right)\left(\gamma_{x}(s)\right) \\
& =\left(\operatorname{Ric}_{f}^{N}\left(\gamma_{x}^{\prime}(s)\right)+\frac{f_{x}^{\prime}(s)^{2}}{N-n}\right)+\left\|\operatorname{Hess} \rho_{\partial M}\right\|^{2}\left(\gamma_{x}(s)\right)-h_{f, x}^{\prime}(s) .
\end{aligned}
$$


From the assumption $\operatorname{Ric}_{f}^{N}\left(\gamma_{x}^{\prime}(s)\right) \geq \kappa$, we deduce

$$
0 \geq \kappa+\frac{f_{x}^{\prime}(s)^{2}}{N-n}+\left\|\operatorname{Hess} \rho_{\partial M}\right\|^{2}\left(\gamma_{x}(s)\right)-h_{f, x}^{\prime}(s) .
$$

By the Cauchy-Schwarz inequality, we have

$$
\left\|\operatorname{Hess} \rho_{\partial M}\right\|^{2}\left(\gamma_{x}(s)\right) \geq \frac{\left(\Delta \rho_{\partial M}\left(\gamma_{x}(s)\right)\right)^{2}}{n-1}=\frac{\left(h_{f, x}(s)-f_{x}^{\prime}(s)\right)^{2}}{n-1} .
$$

Combining (3.4) and (3.5), we see

$$
0 \geq \kappa+\frac{f_{x}^{\prime}(s)^{2}}{N-n}+\frac{\left(h_{f, x}(s)-f_{x}^{\prime}(s)\right)^{2}}{n-1}-h_{f, x}^{\prime}(s)
$$

$$
=\kappa+\frac{(1-N) f_{x}^{\prime}(s)^{2}}{(n-1)(n-N)}+\frac{h_{f, x}(s)^{2}}{n-1}-\left(\frac{2 h_{f, x}(s) f_{x}^{\prime}(s)}{n-1}+h_{f, x}^{\prime}(s)\right) .
$$

In the right hand side of the equation $(3.6)$, by $N \leq 1$, the second term is non-negative. The third one is non-negative. The last one satisfies

$$
\begin{aligned}
\frac{2 h_{f, x}(s) f_{x}^{\prime}(s)}{n-1}+h_{f, x}^{\prime}(s) & =e^{-\frac{2 f_{x}(s)}{n-1}}\left(e^{\frac{2 f_{x}(s)}{n-1}} h_{f, x}(s)\right)^{\prime} \\
& =F_{x}^{-2}(s)\left(F_{x}^{2}(s) h_{f, x}(s)\right)^{\prime} .
\end{aligned}
$$

Hence, we have $0 \geq \kappa-F_{x}^{-2}(s)\left(F_{x}^{2}(s) h_{f, x}(s)\right)^{\prime}$. This implies (3.3).

We see that $F_{x}(s)$ tends to 1 as $s \rightarrow 0$. Furthermore, by Lemma 2.7 , $h_{f, x}(s)$ tends to $H_{f, x}$ as $s \rightarrow 0$. It follows that

$$
F_{x}^{2}(s) h_{f, x}(s)-\kappa \int_{0}^{s} F_{x}^{2}(u) d u \rightarrow H_{f, x}
$$

as $s \rightarrow 0$. By $(3.3)$ and (3.7), for all $s, t \in(0, \tau(x))$ with $s \leq t$

$$
\begin{aligned}
F_{x}^{2}(t) h_{f, x}(t)-\kappa & \int_{0}^{t} F_{x}^{2}(u) d u \\
& \geq F_{x}^{2}(s) h_{f, x}(s)-\kappa \int_{0}^{s} F_{x}^{2}(u) d u \geq H_{f, x} \geq \lambda .
\end{aligned}
$$

Thus, we arrive at 3.2 .

Remark 3.1. Under the same setting as in Lemma 3.1. Wylie [39] has shown a Laplacian comparison inequality for the distance function from a connected component of the boundary that is similar to 3.2 when $\kappa=0$ and $\lambda=0$ (see the proof of Theorem 5.1 in [39]).

Remark 3.2. Assume that for some $t_{0} \in(0, \tau(x))$ the equality in (3.2) holds. Then (3.8) implies that $F_{x}^{2} h_{f, x}=F_{\kappa, \lambda, x}$ and $\left(F_{x}^{2} h_{f, x}\right)^{\prime}=\kappa F_{x}^{2}$ on $\left(0, t_{0}\right)$. Hence, for every $t \in\left(0, t_{0}\right)$, the equality in the Cauchy-Schwarz 
inequality in (3.5) holds; in particular, there exists a function $\varphi$ on $\gamma_{x}\left(\left(0, t_{0}\right)\right)$ such that at each point on $\gamma_{x}\left(\left(0, t_{0}\right)\right)$ we have Hess $\rho_{\partial M}=$ $\varphi g$ on the orthogonal complement of $\nabla \rho_{\partial M}$. Furthermore, for every $t \in\left(0, t_{0}\right)$, the second term and the third one in the right hand side of (3.6) are equal to 0 ; in particular, $(1-N)\left(f_{x}^{\prime}\right)^{2}=0$ on $\left(0, t_{0}\right)$.

From Lemma 3.1, we derive the following:

Lemma 3.2. Take $x \in \partial M$. For $\kappa, \lambda \in \mathbb{R}$ and for $N \in(-\infty, 1]$ we suppose that for all $t \in(0, \tau(x))$ we have $\operatorname{Ric}_{f}^{N}\left(\gamma_{x}^{\prime}(t)\right) \geq \kappa$, and suppose $H_{f, x} \geq \lambda$. Then for all $s, t \in[0, \tau(x))$ with $s \leq t$ we have

$$
\theta_{f}(t, x) \leq e^{-\int_{s}^{t} F_{x}^{-2}(u) F_{\kappa, \lambda, x}(u) d u} \theta_{f}(s, x),
$$

where $\theta_{f}(t, x)$ is defined as (2.1). In particular, if $\kappa$ and $\lambda$ satisfy the subharmonic-condition, then for all $s, t \in[0, \tau(x))$ with $s \leq t$ we have $\theta_{f}(t, x) \leq \theta_{f}(s, x)$.

Proof. By 2.2 and Lemma 3.1, for all $t \in(0, \tau(x))$

$$
\frac{d}{d t} \log \frac{e^{-\int_{0}^{t} F_{x}^{-2}(u) F_{\kappa, \lambda, x}(u) d u}}{\theta_{f}(t, x)}=-F_{x}^{-2}(t) F_{\kappa, \lambda, x}(t)+\Delta_{f} \rho_{\partial M}\left(\gamma_{x}(t)\right) \geq 0 .
$$

It follows that for all $s, t \in[0, \tau(x))$ with $s \leq t$

$$
\frac{\theta_{f}(t, x)}{\theta_{f}(s, x)} \leq \frac{e^{-\int_{0}^{t} F_{x}^{-2}(u) F_{\kappa, \lambda, x}(u) d u}}{e^{-\int_{0}^{s} F_{x}^{-2}(u) F_{\kappa, \lambda, x}(u) d u}} .
$$

Therefore, we have the lemma.

3.2. Equality cases. We recall the following radial curvature equation (see e.g., Theorem 2 in [29]):

Lemma 3.3. Let $\rho$ be a smooth function defined on a domain in $M$ such that $\|\nabla \rho\|=1$. Let $X$ be a parallel vector field along an integral curve of $\nabla \rho$ that is orthogonal to $\nabla \rho$. Then we have

$$
g(R(X, \nabla \rho) \nabla \rho, X)=g\left(\nabla_{\nabla \rho} A_{\nabla \rho} X, X\right)-g\left(A_{\nabla \rho} A_{\nabla \rho} X, X\right),
$$

where $R$ is the curvature tensor induced from $g$, and $A_{\nabla \rho}$ is the shape operator of the level set of $\rho$ toward $\nabla \rho$. In particular, if there exists a function $\varphi$ defined on the domain of the integral curve such that $A_{\nabla \rho} X=-\varphi X$, then we have

$$
g(R(X, \nabla \rho) \nabla \rho, X)=-\left(\varphi^{\prime}+\varphi^{2}\right)\|X\|^{2} .
$$

For the equality case of Lemma 3.1, we have: 
Lemma 3.4. Take $x \in \partial M$. For $\kappa, \lambda \in \mathbb{R}$ and for $N \in(-\infty, 1]$ we suppose that for all $t \in(0, \tau(x))$ we have $\operatorname{Ric}_{f}^{N}\left(\gamma_{x}^{\prime}(t)\right) \geq \kappa$, and suppose $H_{f, x} \geq \lambda$. Choose an orthonormal basis $\left\{e_{x, i}\right\}_{i=1}^{n-1}$ of $T_{x} \partial M$, and let $\left\{Y_{x, i}\right\}_{i=1}^{n-1}$ be the $\partial M$-Jacobi fields along $\gamma_{x}$ with initial conditions $Y_{x, i}(0)=e_{x, i}$ and $Y_{x, i}^{\prime}(0)=-A_{u_{x}} e_{x, i}$. Assume that for some $t_{0} \in$ $(0, \tau(x))$ the equality in (3.2) holds. Then $\kappa=0$ and $\lambda=0$, and for all $i$ we have $Y_{x, i}=F_{x} E_{x, i}$ on $\left[0, t_{0}\right]$, where $\left\{E_{x, i}\right\}_{i=1}^{n-1}$ are the parallel vector fields along $\gamma_{x}$ with initial condition $E_{x, i}(0)=e_{x, i}$. Moreover, if $N \in(-\infty, 1)$, then $f \circ \gamma_{x}$ is constant on $\left[0, t_{0}\right]$; in particular, $Y_{x, i}=E_{x, i}$.

Proof. By the equality assumption, there exists a function $\varphi$ on the set $\gamma_{x}\left(\left(0, t_{0}\right)\right)$ such that at each point on $\gamma_{x}\left(\left(0, t_{0}\right)\right)$ we have Hess $\rho_{\partial M}=\varphi g$ on the orthogonal complement of $\nabla \rho_{\partial M}$ (see Remark 3.2). Put $\varphi_{x}:=$ $\varphi \circ \gamma_{x}$. For each $i$, it holds that

$$
g\left(A_{\nabla \rho_{\partial M}} E_{x, i}, E_{x, i}\right)=-\operatorname{Hess} \rho_{\partial M}\left(E_{x, i}, E_{x, i}\right)=-\varphi_{x} .
$$

It follows that $A_{\nabla \rho_{\partial M}} E_{x, i}=-\varphi_{x} E_{x, i}$. From Lemma 3.3, we derive

$$
R\left(E_{x, i}, \nabla \rho_{\partial M}\right) \nabla \rho_{\partial M}=-\left(\varphi_{x}^{\prime}+\varphi_{x}^{2}\right) E_{x, i} .
$$

Put $f_{x}:=f \circ \gamma_{x}$ and $h_{f, x}:=\left(\Delta_{f} \rho_{\partial M}\right) \circ \gamma_{x}$. By the equality assumption, we have $F_{x}^{2} h_{f, x}=F_{\kappa, \lambda, x}$ on $\left[0, t_{0}\right]$ (see Remark 3.2). Fix $t \in\left[0, t_{0}\right]$. Since $h_{f, x}(t)=F_{x}^{-2}(t) F_{\kappa, \lambda, x}(t)$, we have

$$
\Delta \rho_{\partial M}\left(\gamma_{x}(t)\right)=-f_{x}^{\prime}(t)+F_{x}^{-2}(t) F_{\kappa, \lambda, x}(t) .
$$

On the other hand, from Hess $\rho_{\partial M}=\varphi g$, we deduce $\Delta \rho_{\partial M}\left(\gamma_{x}(t)\right)=$ $-(n-1) \varphi_{x}(t)$. Hence, $\varphi_{x}(t)$ is equal to

$$
(n-1)^{-1}\left(f_{x}(t)-f(x)-\int_{0}^{t} F_{x}^{-2}(s) F_{\kappa, \lambda, x}(s) d s\right)^{\prime} .
$$

Now, define a function $\mathcal{F}_{x}:\left[0, t_{0}\right] \rightarrow(0, \infty)$ by

$$
\mathcal{F}_{x}(t):=e^{\int_{0}^{t} \varphi_{x}(s) d s}=e^{-\frac{\int_{0}^{t} F_{x}^{-2}(s) F_{k, \lambda, x}(s) d s}{n-1}} F_{x}(t) .
$$

Note that if $\kappa=0$ and $\lambda=0$, then $\mathcal{F}_{x}=F_{x}$. By (3.9), a vector field $\tilde{Y}_{x, i}$ along $\left.\gamma_{x}\right|_{\left[0, t_{0}\right]}$ defined by $\tilde{Y}_{x, i}:=\mathcal{F}_{x} E_{x, i}$ is a $\partial M$-Jacobi field along $\left.\gamma_{x}\right|_{\left[0, t_{0}\right]}$ with initial conditions $Y_{x, i}(0)=e_{x, i}$ and $Y_{x, i}^{\prime}(0)=-A_{u_{x}} e_{x, i}$. Therefore, $Y_{x, i}$ coincides with $\tilde{Y}_{x, i}$ on $\left[0, t_{0}\right]$.

From (3.9) and $Y_{x, i}=\mathcal{F}_{x} E_{x, i}$, we derive $R\left(E_{x, i}, \nabla \rho_{\partial M}\right) \nabla \rho_{\partial M}=$ $-\mathcal{F}_{x}^{\prime \prime} \mathcal{F}_{x}^{-1} E_{x, i}$. This implies that for each $t \in\left(0, t_{0}\right)$

$$
\begin{aligned}
\operatorname{Ric}_{g}\left(\gamma_{x}^{\prime}(t)\right) & =-(n-1) \mathcal{F}_{x}^{\prime \prime}(t) \mathcal{F}_{x}^{-1}(t) \\
& =-f_{x}^{\prime \prime}(t)-\frac{f_{x}^{\prime}(t)^{2}}{n-1}+\kappa-\frac{F_{x}^{-4}(t) F_{\kappa, \lambda, x}^{2}(t)}{n-1} ;
\end{aligned}
$$


in particular, $\operatorname{Ric}_{f}^{1}\left(\gamma_{x}^{\prime}(t)\right)=\kappa-(n-1)^{-1} F_{x}^{-4}(t) F_{\kappa, \lambda, x}^{2}(t)$. By the monotonicity of $\operatorname{Ric}_{f}^{N}$ with respect to $N$, we see

$$
\kappa \leq \operatorname{Ric}_{f}^{N}\left(\gamma_{x}^{\prime}(t)\right) \leq \operatorname{Ric}_{f}^{1}\left(\gamma_{x}^{\prime}(t)\right)=\kappa-\frac{F_{x}^{-4}(t) F_{\kappa, \lambda, x}^{2}(t)}{n-1},
$$

and hence $F_{\kappa, \lambda, x}(t)=0$. We obtain $\kappa=0$ and $\lambda=0$, and $Y_{x, i}=F_{x} E_{x, i}$.

Now, we have $(1-N)\left(f_{x}^{\prime}\right)^{2}=0$ on $\left[0, t_{0}\right]$ (see Remark 3.2). If $N$ is smaller than 1 , then $f_{x}^{\prime}=0$ on $\left[0, t_{0}\right]$; in particular, $f_{x}$ is constant on $\left[0, t_{0}\right]$. This completes the proof.

By Lemma 3.4, we have the following:

Lemma 3.5. Take $x \in \partial M$. Let $\kappa \in \mathbb{R}$ and $\lambda \in \mathbb{R}$ satisfy the subharmonic-condition. For $N \in(-\infty, 1]$ we suppose that for all $t \in(0, \tau(x))$ we have $\operatorname{Ric}_{f}^{N}\left(\gamma_{x}^{\prime}(t)\right) \geq \kappa$, and suppose $H_{f, x} \geq \lambda$. We choose an orthonormal basis $\left\{e_{x, i}\right\}_{i=1}^{n-1}$ of $T_{x} \partial M$, and let $\left\{Y_{x, i}\right\}_{i=1}^{n-1}$ be the $\partial M$-Jacobi fields along $\gamma_{x}$ with initial conditions $Y_{x, i}(0)=e_{x, i}$ and $Y_{x, i}^{\prime}(0)=-A_{u_{x}} e_{x, i}$. Assume that for some $t_{0} \in(0, \tau(x))$ we have $\Delta_{f} \rho_{\partial M}\left(\gamma_{x}\left(t_{0}\right)\right)=0$. Then $\kappa=0$ and $\lambda=0$, and for all $i$ we have $Y_{x, i}=F_{x} E_{x, i}$ on $\left[0, t_{0}\right]$, where $\left\{E_{x, i}\right\}_{i=1}^{n-1}$ are the parallel vector fields along $\gamma_{x}$ with initial condition $E_{x, i}(0)=e_{x, i}$. Moreover, if $N \in(-\infty, 1)$, then $f \circ \gamma_{x}$ is constant on $\left[0, t_{0}\right]$; in particular, $Y_{x, i}=E_{x, i}$.

Proof. The assumption $\Delta_{f} \rho_{\partial M}\left(\gamma_{x}\left(t_{0}\right)\right)=0$ implies that the equality in (3.2) holds. Lemma 3.4 leads to the lemma.

3.3. Distributions. From Lemma 3.1, we derive the following:

Lemma 3.6. Take $x \in \partial M$. Let $p \in(1, \infty)$, and let $\kappa \in \mathbb{R}$ and $\lambda \in \mathbb{R}$ satisfy the subharmonic-condition. For $N \in(-\infty, 1]$ we suppose that for all $t \in(0, \tau(x))$ we have $\operatorname{Ric}_{f}^{N}\left(\gamma_{x}^{\prime}(t)\right) \geq \kappa$, and suppose $H_{f, x} \geq \lambda$. Let $\varphi:[0, \infty) \rightarrow \mathbb{R}$ be a monotone increasing smooth function. Then for all $t \in(0, \tau(x))$

$$
\Delta_{f, p}\left(\varphi \circ \rho_{\partial M}\right)\left(\gamma_{x}(t)\right) \geq-\left(\left(\varphi^{\prime}\right)^{p-1}\right)^{\prime}(t) .
$$

Proof. For all $t \in(0, \tau(x))$ we see

$$
\Delta_{f, p}\left(\varphi \circ \rho_{\partial M}\right)\left(\gamma_{x}(t)\right)=-\left(\left(\varphi^{\prime}\right)^{p-1}\right)^{\prime}(t)+\Delta_{f, 2} \rho_{\partial M}\left(\gamma_{x}(t)\right) \varphi^{\prime}(t)^{p-1} .
$$

This together with Lemma 3.1 implies 3.10.

Remark 3.3. The equality case in Lemma 3.6 corresponds to that in Lemma 3.1 (see Lemma 3.5).

From Lemma 3.6, we deduce the following: 
Proposition 3.7. Let $p \in(1, \infty)$, and let $\kappa \in \mathbb{R}$ and $\lambda \in \mathbb{R}$ satisfy the subharmonic-condition. For $N \in(-\infty, 1]$ we suppose $\operatorname{Ric}_{f, M}^{N} \geq \kappa$, and $H_{f, \partial M} \geq \lambda$. For a monotone increasing smooth function $\varphi:[0, \infty) \rightarrow$ $\mathbb{R}$, we put $\Phi:=\varphi \circ \rho_{\partial M}$. Then

$$
\Delta_{f, p} \Phi \geq-\left(\left(\varphi^{\prime}\right)^{p-1}\right)^{\prime} \circ \rho_{\partial M}
$$

in a distribution sense on $M$. More precisely, for every non-negative smooth function $\psi: M \rightarrow \mathbb{R}$ whose support is compact and contained in Int $M$, we have

$$
\int_{M}\|\nabla \Phi\|^{p-2} g(\nabla \psi, \nabla \Phi) d m_{f} \geq \int_{M} \psi\left(-\left(\left(\varphi^{\prime}\right)^{p-1}\right)^{\prime} \circ \rho_{\partial M}\right) d m_{f} .
$$

Proof. By Lemma 2.3, there exists a sequence $\left\{\Omega_{k}\right\}_{k \in \mathbb{N}}$ of closed subsets of $M$ such that for every $k$, the set $\partial \Omega_{k}$ is a smooth hypersurface in $M$ satisfying the following: (1) for all $k_{1}, k_{2} \in \mathbb{N}$ with $k_{1}<k_{2}$, we have $\Omega_{k_{1}} \subset \Omega_{k_{2}} ;(2) M \backslash$ Cut $\partial M=\bigcup_{k} \Omega_{k} ;(3) \partial \Omega_{k} \cap \partial M=\partial M$ for all $k ;(4)$ for each $k$, on $\partial \Omega_{k} \backslash \partial M$, there exists the unit outer normal vector field $\nu_{k}$ for $\Omega_{k}$ with $g\left(\nu_{k}, \nabla \rho_{\partial M}\right) \geq 0$. For the canonical Riemannian volume measure $\operatorname{vol}_{k}$ on $\partial \Omega_{k} \backslash \partial M$, put $m_{f, k}:=e^{-f \mid \partial \Omega_{k} \backslash \partial M} \operatorname{vol}_{k}$. Let $\psi: M \rightarrow$ $\mathbb{R}$ be a non-negative smooth function whose support is compact and contained in Int $M$. By the Green formula, and by $\partial \Omega_{k} \cap \partial M=\partial M$,

$$
\begin{aligned}
& \int_{\Omega_{k}} \| \nabla \Phi \|^{p-2} g(\nabla \psi, \nabla \Phi) d m_{f} \\
& \quad=\int_{\Omega_{k}} \psi \Delta_{f, p} \Phi d m_{f}+\int_{\partial \Omega_{k} \backslash \partial M}\|\nabla \Phi\|^{p-2} \psi g\left(\nu_{k}, \nabla \Phi\right) d m_{f, k} .
\end{aligned}
$$

From Lemma 3.6 and $g\left(\nu_{k}, \nabla \rho_{\partial M}\right) \geq 0$, we derive

$$
\int_{\Omega_{k}}\|\nabla \Phi\|^{p-2} g(\nabla \psi, \nabla \Phi) d m_{f} \geq \int_{\Omega_{k}} \psi\left(-\left(\left(\varphi^{\prime}\right)^{p-1}\right)^{\prime} \circ \rho_{\partial M}\right) d m_{f} .
$$

Letting $k \rightarrow \infty$, we have the proposition.

Remark 3.4. In Proposition 3.7, we assume that the equality in (3.11) holds. Then for every $x \in \partial M$, and for every $t \in(0, \tau(x))$, the equality in (3.10) also holds. The equality case in Proposition 3.7 corresponds to that in Lemma 3.6 (see Remark 3.3).

Remark 3.5. Perales [28] has shown a Laplacian comparison inequality for the distance function from the boundary in a barrier sense for manifolds with boundary of non-negative Ricci curvature. We can prove that the Laplacian comparison inequality for $\rho_{\partial M}$ in Lemma 3.1 globally holds on $M$ in a barrier sense. 


\section{VOLUME COMPARISONS}

Let $M$ be an $n$-dimensional, connected complete Riemannian manifold with boundary with Riemannian metric $g$, and let $f: M \rightarrow \mathbb{R}$ be a smooth function.

4.1. Absolute volume comparisons. We have the following absolute volume comparison inequality of Heintze-Karcher type:

Lemma 4.1. Suppose that $\partial M$ is compact. For $\kappa, \lambda \in \mathbb{R}$, and for $N \in(-\infty, 1]$ we suppose $\operatorname{Ric}_{f, M}^{N} \geq \kappa$, and $H_{f, \partial M} \geq \lambda$. Then for all $r \in(0, \infty)$ we have

$$
m_{f}\left(B_{r}(\partial M)\right) \leq \int_{\partial M} \int_{0}^{\min \{r, \tau(x)\}} e^{-\int_{0}^{t} F_{x}^{-2}(u) F_{\kappa, \lambda, x}(u) d u} d t d m_{f, \partial M},
$$

where $F_{\kappa, \lambda, x}$ is the function defined as (3.1). In particular, if $\kappa$ and $\lambda$ satisfy the subharmonic-condition, then for all $r \in(0, \infty)$ we have $m_{f}\left(B_{r}(\partial M)\right) \leq r m_{f, \partial M}(\partial M)$, and hence 1.7 .

Proof. Define a function $\tilde{\theta}:[0, \infty) \times \partial M \rightarrow \mathbb{R}$ by

$$
\tilde{\theta}(t, x):= \begin{cases}e^{-\int_{0}^{t} F_{x}^{-2}(u) F_{\kappa, \lambda, x}(u) d u} & \text { if } t<\tau(x), \\ 0 & \text { if } t \geq \tau(x) .\end{cases}
$$

By Lemma 3.2, for all $x \in \partial M$ and $t \in(0, \infty)$ we see $\bar{\theta}_{f}(t, x) \leq$ $\tilde{\theta}(t, x) e^{-f(x)}$, where $\bar{\theta}_{f}$ is the function defined as 2.3 . Integrate the both sides of the inequality over $(0, r)$ with respect to $t$, and then do that over $\partial M$ with respect to $x$. From Lemma 2.8, we deduce

$$
m_{f}\left(B_{r}(\partial M)\right) \leq \int_{\partial M} \int_{0}^{r} \tilde{\theta}(t, x) d t d m_{f, \partial M} .
$$

This implies the lemma.

Remark 4.1. Under a lower $N$-weighted Ricci curvature bound, Bayle [3] has stated an inequality of Heintze-Karcher type without proof in the case of $N \in[n, \infty)$. Morgan [24] has proved it in the case of $N=\infty$, and Milman [23] has done in the case of $N \in(-\infty, 1)$.

4.2. Relative volume comparisons. We have the following relative volume comparison theorem of Bishop-Gromov type:

Theorem 4.2. Let $M$ be a connected complete Riemannian manifold with boundary, and let $f: M \rightarrow \mathbb{R}$ be a smooth function. Suppose that $\partial M$ is compact. Let $\kappa \in \mathbb{R}$ and $\lambda \in \mathbb{R}$ satisfy the subharmoniccondition. For $N \in(-\infty, 1]$ we suppose $\operatorname{Ric}_{f, M}^{N} \geq \kappa$, and $H_{f, \partial M} \geq \lambda$. 
Then for all $r, R \in(0, \infty)$ with $r \leq R$

$$
\frac{m_{f}\left(B_{R}(\partial M)\right)}{m_{f}\left(B_{r}(\partial M)\right)} \leq \frac{R}{r}
$$

Proof. Lemma 3.2 implies that for all $s, t \in[0, \infty)$ with $s \leq t$ we have $\bar{\theta}_{f}(t, x) \leq \theta_{f}(s, x)$, where $\bar{\theta}_{f}$ is the function defined as $(2.3)$. By integrating the both sides over $(0, r)$ with respect to $s$, and then doing that over $(r, R)$ with respect to $t$, we see

$$
r \int_{r}^{R} \bar{\theta}_{f}(t, x) d t \leq(R-r) \int_{0}^{r} \bar{\theta}_{f}(s, x) d s .
$$

From Lemma 2.8, we derive

$$
\frac{m_{f}\left(B_{R}(\partial M)\right)}{m_{f}\left(B_{r}(\partial M)\right)}=1+\frac{\int_{\partial M} \int_{r}^{R} \bar{\theta}_{f}(t, x) d t d \operatorname{vol}_{h}}{\int_{\partial M} \int_{0}^{r} \bar{\theta}_{f}(s, x) d s d \operatorname{vol}_{h}} \leq 1+\frac{R-r}{r}=\frac{R}{r} .
$$

This proves the theorem.

When $\kappa=0$ and $\lambda=0$, Theorem 4.2 has been proved in the unweighted case in [32], and in the usual weighted case in [33].

Remark 4.2. In 32, the author has proved a measure contraction inequality around the boundary in the unweighted case. We can prove a similar measure contraction inequality in our setting. The measure contraction inequality enables us to give another proof of Theorem 4.2.

4.3. Volume growth rigidity. We show the following:

Lemma 4.3. Suppose that $\partial M$ is compact. Let $\kappa \in \mathbb{R}$ and $\lambda \in \mathbb{R}$ satisfy the subharmonic-condition. For $N \in(-\infty, 1]$ we suppose $\operatorname{Ric}_{f, M}^{N} \geq$ $\kappa$, and $H_{f, \partial M} \geq \lambda$. Assume that there exists $R \in(0, \infty)$ such that for every $r \in(0, R]$ the equality in (4.1) holds. Then $\tau \geq R$ on $\partial M$.

Proof. The proof will be done by contradiction. Suppose that there exists $x_{0} \in \partial M$ such that $\tau\left(x_{0}\right)<R$. Put $t_{0}:=\tau\left(x_{0}\right)$. Take $\epsilon \in(0, \infty)$ with $t_{0}+\epsilon<R$. By the continuity of $\tau$, there exists a closed geodesic ball $B$ in $\partial M$ centered at $x_{0}$ such that $\tau$ is smaller than or equal to $t_{0}+\epsilon$ on $B$. Using Lemma 3.2 , we see

$m_{f}\left(B_{R}(\partial M)\right) \leq R m_{f, \partial M}(\partial M \backslash B)+\left(t_{0}+\epsilon\right) m_{f, \partial M}(B)<R m_{f, \partial M}(\partial M)$.

On the other hand, $m_{f}\left(B_{R}(\partial M)\right) / m_{f, \partial M}(\partial M)$ is equal to $R$. This is a contradiction.

Suppose that $\partial M$ is compact. Let $\kappa \in \mathbb{R}$ and $\lambda \in \mathbb{R}$ satisfy the subharmonic-condition. For $N \in(-\infty, 1]$ we suppose $\operatorname{Ric}_{f, M}^{N} \geq \kappa$, and $H_{f, \partial M} \geq \lambda$. Assume that there exists $R \in(0, \infty)$ such that for every 
$r \in(0, R]$ the equality in 4 .1) holds. Then for each $r \in(0, R)$ the level set $\rho_{\partial M}^{-1}(r)$ is an $(n-1)$-dimensional submanifold of $M$ (see Lemma 4.3). In particular, $\left(B_{r}(\partial M), g\right)$ is an $n$-dimensional (not necessarily, connected) complete Riemannian manifold with boundary. We denote by $d_{B_{r}(\partial M)}$ and by $d_{[0, r] \times_{F} \partial M}$ the Riemannian distances on $\left(B_{r}(\partial M), g\right)$ and on $[0, r] \times_{F} \partial M$, respectively, where $[0, r] \times_{F} \partial M$ is the twisted product space $\left([0, r] \times \partial M, d t^{2}+F_{x}^{2}(t) h\right)$.

Lemma 4.4. Suppose that $\partial M$ is compact. Let $\kappa \in \mathbb{R}$ and $\lambda \in$ $\mathbb{R}$ satisfy the subharmonic-condition. For $N \in(-\infty, 1]$ we suppose $\operatorname{Ric}_{f, M}^{N} \geq \kappa$, and $H_{f, \partial M} \geq \lambda$. Assume that there exists $R \in(0, \infty)$ such that for every $r \in(0, R]$ the equality in (4.1) holds. Then for each $r \in(0, R)$ the metric space $\left(B_{r}(\partial M), d_{B_{r}(\partial M)}\right)$ is isometric to $\left([0, r] \times_{F} \partial M, d_{[0, r] \times_{F} \partial M}\right)$. Moreover, if $N \in(-\infty, 1)$, then for every $x \in \partial M$ the function $f \circ \gamma_{x}$ is constant on $[0, r]$; in particular, $\left(B_{r}(\partial M), d_{B_{r}(\partial M)}\right)$ is isometric to $\left([0, r] \times \partial M, d_{[0, r] \times \partial M}\right)$.

Proof. Since each connected component of $\partial M$ one-to-one corresponds to the connected component of $B_{r}(\partial M)$, it suffices to consider the case where $B_{r}(\partial M)$ is connected. For each $x \in \partial M$ we choose an orthonormal basis $\left\{e_{x, i}\right\}_{i=1}^{n-1}$ of $T_{x} \partial M$. Let $\left\{Y_{x, i}\right\}_{i=1}^{n-1}$ be the $\partial M$-Jacobi fields along $\gamma_{x}$ with initial conditions $Y_{x, i}(0)=e_{x, i}$ and $Y_{x, i}^{\prime}(0)=$ $-A_{u_{x}} e_{x, i}$. Since the equality in (4.1) holds, for all $t \in[0, r]$ we see $\theta_{f}(t, x)=\theta_{f}(r, x)$. By (2.2), for all $t \in(0, r]$ we see $\Delta_{f} \rho_{\partial M}\left(\gamma_{x}(t)\right)=0$. Lemma 3.5 implies that we have $\kappa=0$ and $\lambda=0$, and for all $i$ we have $Y_{x, i}=F_{x} E_{x, i}$ on $[0, r]$, where $\left\{E_{x, i}\right\}_{i=1}^{n-1}$ are the parallel vectors field along $\gamma_{x}$ with initial condition $E_{x, i}(0)=e_{x, i}$; moreover, if $N \in(-\infty, 1)$, then $f \circ \gamma_{x}$ is constant on $[0, r]$. Define a map $\Phi:[0, r] \times \partial M \rightarrow B_{r}(\partial M)$ by $\Phi(t, x):=\gamma_{x}(t)$. For each $p \in(0, r) \times$ $\partial M$ the differential map $D\left(\left.\Phi\right|_{(0, r) \times \partial M)_{p}}\right.$ sends an orthonormal basis of $T_{p}([0, r] \times \partial M)$ to that of $T_{\Phi(p)} B_{r}(\partial M)$, and for each $x \in\{0, r\} \times \partial M$

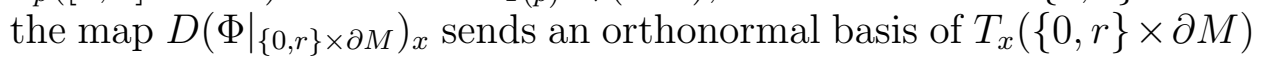
to that of $T_{\Phi(x)} \partial\left(B_{r}(\partial M)\right)$. Hence, $\Phi$ is a Riemannian isometry with boundary from $[0, r] \times_{F} \partial M$ to $B_{r}(\partial M)$.

Now, we are in a position to prove Theorem 1.1 and Corollary 1.2 .

Proof of Theorem 1.1. Suppose that $\partial M$ is compact. Let $\kappa \in \mathbb{R}$ and $\lambda \in \mathbb{R}$ satisfy the subharmonic-condition. For $N \in(-\infty, 1]$ we suppose $\operatorname{Ric}_{f, M}^{N} \geq \kappa$, and $H_{f, \partial M} \geq \lambda$. Furthermore, we assume (1.6). By Lemma 4.1 and Theorem 4.2 , for every $R \in(0, \infty)$, and for every $r \in(0, R]$,

$$
\frac{m_{f}\left(B_{R}(\partial M)\right)}{R}=\frac{m_{f}\left(B_{r}(\partial M)\right)}{r}=m_{f, \partial M}(\partial M) ;
$$


in particular, the equality in 4.1 holds. From Lemma 4.3, we deduce $\tau=\infty$ on $\partial M$. We see Cut $\partial M=\emptyset$, and hence $\partial M$ is connected. Take a sequence $\left\{r_{i}\right\}$ with $r_{i} \rightarrow \infty$. By Lemma 4.4, for every $i$ there exists a Riemannian isometry $\Phi_{i}:\left[0, r_{i}\right] \times \partial M \rightarrow B_{r_{i}}(\partial M)$ with boundary from $\left[0, r_{i}\right] \times_{F} \partial M$ to $B_{r_{i}}(\partial M)$ defined by $\Phi_{i}(t, x):=\gamma_{x}(t)$. Moreover, if $N \in(-\infty, 1)$, then for each $x \in \partial M$ the function $f \circ \gamma_{x}$ is constant on $\left[0, r_{i}\right]$. Since Cut $\partial M=\emptyset$, we obtain a Riemannian isometry $\Phi$ : $[0, \infty) \times \partial M \rightarrow M$ with boundary from $[0, \infty) \times{ }_{F} \partial M$ to $M$ defined by $\Phi(t, x):=\gamma_{x}(t)$ such that $\left.\Phi\right|_{\left[0, r_{i}\right] \times \partial M}=\Phi_{i}$ for all $i$. Furthermore, if $N \in(-\infty, 1)$, then $f \circ \gamma_{x}$ is constant on $[0, \infty)$. Thus, we complete the proof of Theorem 1.1 .

Proof of Corollary 1.2. Combining Theorem 1.1 and Proposition 2.9. we conclude Corollary 1.2 .

\section{Splitting TheOREMS}

Let $M$ be an $n$-dimensional, connected complete Riemannian manifold with boundary with Riemannian metric $g$, and let $f: M \rightarrow \mathbb{R}$ be a smooth function.

5.1. Basic splitting. Let $\varphi: M \rightarrow \mathbb{R}$ be a continuous function, and let $U$ be a domain contained in Int $M$. For $p \in U$, and for a function $\psi$ defined on an open neighborhood of $p$, we say that $\psi$ is a support function of $\varphi$ at $p$ if we have $\psi(p)=\varphi(p)$ and $\psi \leq \varphi$. We say that $\varphi$ is $f$-subharmonic on $U$ if for every $p \in U$, and for every $\epsilon \in(0, \infty)$, there exists a smooth, support function $\psi_{p, \epsilon}$ of $\varphi$ at $p$ such that $\Delta_{f} \psi_{p, \epsilon}(p) \leq \epsilon$.

We recall the following maximal principle of Calabi type (see e.g., [6], and Lemma 2.4 in [10]).

Lemma 5.1. Let $U$ be a domain contained in Int $M$. If a $f$-subharmonic function on $U$ takes the maximal value at a point in $U$, then it must be constant on $U$.

Wylie [39] has proved a subharmonicity of Busemann functions on manifolds without boundary (see Lemma 3.4 in [39]). In our case, under an assumption concerning asymptotes for a ray defined in Subsection 2.4, the subharmonicity holds in the following form:

Lemma $5.2([39])$. Assume $\sup f(M)<\infty$. For $N \in(-\infty, 1]$ we suppose $\operatorname{Ric}_{f, M}^{N} \geq 0$. Let $\gamma:[0, \infty) \rightarrow M$ be a ray that lies in $\operatorname{Int} M$, and let $U$ be a domain contained in Int $M$ such that for each $p \in U$, there exists an asymptote for $\gamma$ from $p$ that lies in Int $M$. Then the Busemann function $b_{\gamma}$ of $\gamma$ is $f$-subharmonic on $U$. 
Now, we prove Theorem 1.3 and Corollary 1.4 .

Proof of Theorem 1.3. Assume $\sup f(M)<\infty$. For $N \in(-\infty, 1]$ we suppose $\operatorname{Ric}_{f, M}^{N} \geq 0$, and $H_{f, \partial M} \geq 0$. Suppose that for some $x_{0} \in \partial M$ we have $\tau\left(x_{0}\right)=\infty$.

For the connected component $\partial M_{0}$ of $\partial M$ containing $x_{0}$, put

$$
\Omega:=\left\{y \in \partial M_{0} \mid \tau(y)=\infty\right\} .
$$

By the continuity of $\tau$, the set $\Omega$ is a non-empty closed subset of $\partial M_{0}$.

We show the openness of $\Omega$ in $\partial M_{0}$. Fix $y_{0} \in \Omega$. Take $l \in(0, \infty)$, and put $p_{0}:=\gamma_{y_{0}}(l)$. There exists an open neighborhood $U$ of $p_{0}$ in Int $M$ contained in $D_{\partial M}$. Taking $U$ smaller, we may assume that for every $q \in U$ the unique foot point on $\partial M$ of $q$ belongs to $\partial M_{0}$. By Lemma 2.5. there exists $\epsilon \in(0, \infty)$ such that for all $q \in B_{\epsilon}\left(p_{0}\right)$, all asymptotes for $\gamma_{y_{0}}$ from $q$ lie in Int $M$. We may assume $U \subset B_{\epsilon}\left(p_{0}\right)$. By Lemma 5.2, $b_{\gamma_{y_{0}}}$ is $f$-subharmonic on $U$, and by Lemma 3.1. $\Delta_{f} \rho_{\partial M} \geq 0$ on $U$. Hence, $b_{\gamma_{y_{0}}}-\rho_{\partial M}$ is $f$-subharmonic on $U$. The function $b_{\gamma_{y_{0}}}-\rho_{\partial M}$ takes the maximal value 0 at $p_{0}$. Lemma 5.1 implies that $b_{\gamma_{y_{0}}}=\rho_{\partial M}$ on $U$. From Lemma 2.4, it follows that $\Omega$ is open in $\partial M_{0}$.

Since $\partial M_{0}$ is a connected component of $\partial M$, we have $\Omega=\partial M_{0}$. By Lemma 2.2, $\partial M$ is connected and Cut $\partial M=\emptyset$. The equality in Lemma 3.1 holds on Int $M$. For each $x \in \partial M$ we choose an orthonormal basis $\left\{e_{x, i}\right\}_{i=1}^{n-1}$ of $T_{x} \partial M$. Let $\left\{Y_{x, i}\right\}_{i=1}^{n-1}$ be the $\partial M$-Jacobi fields along $\gamma_{x}$ with initial conditions $Y_{x, i}(0)=e_{x, i}$ and $Y_{x, i}^{\prime}(0)=-A_{u_{x}} e_{x, i}$. By Lemma 3.4, for all $i$ we have $Y_{x, i}=F_{x} E_{x, i}$ on $[0, \infty)$, where $\left\{E_{x, i}\right\}$ are the parallel vector fields along $\gamma_{x}$ with initial condition $E_{x, i}(0)=e_{x, i}$. Moreover, if $N \in(-\infty, 1)$, then $f \circ \gamma_{x}$ is constant on $[0, \infty)$. Define a map $\Phi:[0, \infty) \times \partial M \rightarrow M$ by $\Phi(t, x):=\gamma_{x}(t)$. For every $p \in$ $(0, \infty) \times \partial M$ the differential map $D\left(\left.\Phi\right|_{(0, \infty) \times \partial M}\right)_{p}$ sends an orthonormal basis of $T_{p}((0, \infty) \times \partial M)$ to that of $T_{\Phi(p)} M$, and for every $x \in\{0\} \times \partial M$ the map $D\left(\left.\Phi\right|_{\{0\} \times \partial M}\right)_{x}$ sends an orthonormal basis of $T_{x}(\{0\} \times \partial M)$ to that of $T_{\Phi(x)} \partial M$. Then $\Phi$ is a Riemannian isometry with boundary from $[0, \infty) \times_{F} \partial M$ to $M$. This proves Theorem 1.3 .

Proof of Corollary 1.4. From Theorem 1.3 and Proposition 2.9, we derive Corollary 1.4 .

Lemma 2.1 and the continuity of $\tau$ imply that if $M$ is non-compact and $\partial M$ is compact, then for some $x \in \partial M$ we have $\tau(x)=\infty$. We have the following corollary of Theorem 1.3 .

Corollary 5.3. Let $M$ be a connected complete Riemannian manifold with boundary, and let $f: M \rightarrow \mathbb{R}$ be a smooth function such that 
$\sup f(M)<\infty$. For $N \in(-\infty, 1]$ we suppose $\operatorname{Ric}_{f, M}^{N} \geq 0$, and $H_{f, \partial M} \geq$ 0 . If $M$ is non-compact and $\partial M$ is compact, then $\left(M, d_{M}\right)$ is isometric to $\left([0, \infty) \times_{F} \partial M, d_{[0, \infty) \times_{F} \partial M}\right)$. Moreover, if $N \in(-\infty, 1)$, then for every $x \in \partial M$ the function $f \circ \gamma_{x}$ is constant on $[0, \infty)$; in particular, $\left(M, d_{M}\right)$ is isometric to $\left([0, \infty) \times \partial M, d_{[0, \infty) \times \partial M}\right)$.

5.2. Weighted Ricci curvature on the boundary. For $x \in \partial M$, we recall that $u_{x}$ denotes the unit inner normal vector on $\partial M$ at $x$.

The following seems to be well-known, especially in a submanifold setting (see e.g., Proposition 9.36 in [4], and Lemma 5.4 in [32]).

Lemma 5.4. Take $x \in \partial M$, and a unit vector $u$ in $T_{x} \partial M$. Choose an orthonormal basis $\left\{e_{x, i}\right\}_{i=1}^{n-1}$ of $T_{x} \partial M$ with $e_{x, 1}=u$. Then we have

$$
\operatorname{Ric}_{h}(u)=\operatorname{Ric}_{g}(u)-K_{g}\left(u_{x}, u\right)+\operatorname{trace} A_{S(u, u)}-\sum_{i=1}^{n-1}\left\|S\left(u, e_{x, i}\right)\right\|^{2},
$$

where $h$ is the induced Riemannian metric on $\partial M$, and $K_{g}\left(u_{x}, u\right)$ is the sectional curvature at $x$ in $(M, g)$ determined by $u_{x}$ and $u$.

By using Lemma 5.4, we have the following:

Lemma 5.5. Take $x \in \partial M$, and a unit vector $u$ in $T_{x} \partial M$. Choose an orthonormal basis $\left\{e_{x, i}\right\}_{i=1}^{n-1}$ of $T_{x} \partial M$ with $e_{x, 1}=u$. Then for all $N \in(-\infty, \infty)$ we have

$$
\begin{aligned}
\operatorname{Ric}_{\left.f\right|_{\partial M}}^{N-1}(u) & =\operatorname{Ric}_{f}^{N}(u)+g\left((\nabla f)_{x}, u_{x}\right) g\left(S(u, u), u_{x}\right) \\
& -K_{g}\left(u_{x}, u\right)+\operatorname{trace} A_{S(u, u)}-\sum_{i=1}^{n-1}\left\|S\left(u, e_{x, i}\right)\right\|^{2},
\end{aligned}
$$

where $K_{g}\left(u_{x}, u\right)$ is the sectional curvature at $x$ in $(M, g)$ determined by $u_{x}$ and $u$.

Proof. First, we assume $N \neq n$. We see

$$
\begin{aligned}
h\left(\left(\nabla\left(\left.f\right|_{\partial M}\right)\right)_{x}, u\right) & =g\left((\nabla f)_{x}, u\right), \\
\operatorname{Hess}\left(\left.f\right|_{\partial M}\right)(u, u) & =\operatorname{Hess} f(u, u)+g\left((\nabla f)_{x}, u_{x}\right) g\left(S(u, u), u_{x}\right),
\end{aligned}
$$

where $h$ is the induced Riemannian metric on $\partial M$. Hence, we have

$$
\begin{aligned}
& \operatorname{Ric}_{\left.f\right|_{\partial M}}^{N-1}(u)=\operatorname{Ric}_{h}(u)+\operatorname{Hess}\left(\left.f\right|_{\partial M}\right)(u, u)-\frac{h\left(\left(\nabla\left(\left.f\right|_{\partial M}\right)\right)_{x}, u\right)^{2}}{(N-1)-(n-1)} \\
= & \operatorname{Ric}_{h}(u)+\operatorname{Hess} f(u, u)+g\left((\nabla f)_{x}, u_{x}\right) g\left(S(u, u), u_{x}\right)-\frac{g\left((\nabla f)_{x}, u\right)^{2}}{N-n} .
\end{aligned}
$$

From Lemma 5.4, we derive 5.1. 
Next, we assume $N=n$. If $f$ is constant, then $\operatorname{Ric}_{\left.f\right|_{\partial M}}^{N-1}(u)=\operatorname{Ric}_{h}(u)$ and $\operatorname{Ric}_{f}^{N}(u)=\operatorname{Ric}_{g}(u)$, and hence Lemma 5.4 implies 5.1). If $f$ is not constant, then both the left hand side of (5.1) and the right hand side are equal to $-\infty$. Therefore, we complete the proof.

From Lemma 5.5, we derive the following:

Lemma 5.6. Take $x \in \partial M$, and a unit vector $u$ in $T_{x} \partial M$. If $\left(M, d_{M}\right)$ is isometric to $\left([0, \infty) \times_{F} \partial M, d_{[0, \infty) \times_{F} \partial M}\right)$, then for all $N \in(-\infty, \infty)$

$$
\operatorname{Ric}_{\left.f\right|_{\text {aM }}}^{N-1}(u)=\operatorname{Ric}_{f}^{N}(u)+\frac{\operatorname{Hess} f\left(u_{x}, u_{x}\right)}{n-1} .
$$

Proof. Choose an orthonormal basis $\left\{e_{x, i}\right\}_{i=1}^{n-1}$ of $T_{x} \partial M$ with $e_{x, 1}=u$. Let $\left\{Y_{x, i}\right\}_{i=1}^{n-1}$ be the $\partial M$-Jacobi fields along $\gamma_{x}$ with initial conditions $Y_{x, i}(0)=e_{x, i}$ and $Y_{x, i}^{\prime}(0)=-A_{u_{x}} e_{x, i}$. Since $\left(M, d_{M}\right)$ is isometric to $\left([0, \infty) \times_{F} \partial M, d_{[0, \infty) \times_{F} \partial M}\right)$, there exists a Riemannian isometry with boundary from $M$ to $[0, \infty) \times_{F} \partial M$. In particular, for all $i$ we see $Y_{x, i}=F_{x} E_{x, i}$, where $\left\{E_{x, i}\right\}_{i=1}^{n-1}$ are the parallel vector fields along $\gamma_{x}$ with initial condition $E_{x, i}(0)=e_{x, i}$. Hence, for all $i$

$$
A_{u_{x}} e_{x, i}=-Y_{x, i}^{\prime}(0)=-\frac{g\left((\nabla f)_{x}, u_{x}\right)}{n-1} e_{x, i} .
$$

By (5.2), for all $i \neq 1$ we have $S\left(u, e_{x, i}\right)=0_{x}$, and we have

$$
S(u, u)=-\frac{g\left((\nabla f)_{x}, u_{x}\right)}{n-1} u_{x}, \quad \operatorname{trace} A_{S(u, u)}=\frac{g\left((\nabla f)_{x}, u_{x}\right)^{2}}{n-1} .
$$

Furthermore, the sectional curvature $K_{g}\left(u_{x}, u\right)$ at $x$ in $(M, g)$ determined by $u_{x}$ and $u$ satisfies

$$
K_{g}\left(u_{x}, u\right)=-g\left(Y_{x, 1}^{\prime \prime}(0), u\right)=-\left(\frac{\operatorname{Hess} f\left(u_{x}, u_{x}\right)}{n-1}+\left(\frac{g\left((\nabla f)_{x}, u_{x}\right)}{n-1}\right)^{2}\right) .
$$

By Lemma 5.5, and by (5.3) and (5.4), we see

$$
\begin{aligned}
\operatorname{Ric}_{\left.f\right|_{\partial M}}^{N-1}(u) & =\operatorname{Ric}_{f}^{N}(u)-\frac{g\left((\nabla f)_{x}, u_{x}\right)^{2}}{n-1}+\frac{\operatorname{Hess} f\left(u_{x}, u_{x}\right)}{n-1} \\
& +\left(\frac{g\left((\nabla f)_{x}, u_{x}\right)}{n-1}\right)^{2}+\frac{g\left((\nabla f)_{x}, u_{x}\right)^{2}}{n-1}-\left(\frac{g\left((\nabla f)_{x}, u_{x}\right)}{n-1}\right)^{2} \\
& =\operatorname{Ric}_{f}^{N}(u)+\frac{\operatorname{Hess} f\left(u_{x}, u_{x}\right)}{n-1} .
\end{aligned}
$$

This completes the proof. 
5.3. Multi-splitting. Let $M_{0}$ be a connected complete Riemannian manifold (without boundary). A normal geodesic $\gamma: \mathbb{R} \rightarrow M_{0}$ is said to be a line if for all $s, t \in \mathbb{R}$ we have $d_{M_{0}}(\gamma(s), \gamma(t))=|s-t|$.

Wylie [39] has proved the following splitting theorem of CheegerGromoll type (see Theorem 1.2 and Corollary 1.3 in [39]):

Theorem 5.7 ([39). Let $M_{0}$ be a connected complete Riemannian manifold, and let $f: M_{0} \rightarrow \mathbb{R}$ be a smooth function bounded from above. For $N \in(-\infty, 1]$ we suppose $\operatorname{Ric}_{f, M_{0}}^{N} \geq 0$. If $M_{0}$ contains a line, then there exists a connected complete Riemannian manifold $N_{0}$ such that $M_{0}$ is isometric to a warped product space over $\mathbb{R} \times N_{0}$. Moreover, if $N \in(-\infty, 1)$, then $M_{0}$ is isometric to the standard product $\mathbb{R} \times N_{0}$.

Remark 5.1. For manifolds of non-negative $N$-weighted Ricci curvature, Lichnerowicz [19] has generalized the Cheeger-Gromoll splitting theorem in the case where $N=\infty$ and $f$ is bounded. Fang, Li and Zhang [10] have done in the case where $N \in[n, \infty)$, and in the case where $N=\infty$ and $f$ is bounded above.

We obtain the following corollary of Theorem 1.3 .

Corollary 5.8. Let $M$ be an $n$-dimensional, connected complete Riemannian manifold with boundary, and let $f: M \rightarrow \mathbb{R}$ be a smooth function such that $\sup f(M)<\infty$. For $N \in(-\infty, 1)$ we suppose $\operatorname{Ric}_{f, M}^{N} \geq 0$, and $H_{f, \partial M} \geq 0$. Assume that for some $x_{0} \in \partial M$ we have $\tau\left(x_{0}\right)=\infty$. Then there exist $k \in\{0, \ldots, n-1\}$ and an $(n-1-k)$ dimensional, connected complete Riemannian manifold $N_{0}$ containing no line such that $\left(\partial M, d_{\partial M}\right)$ is isometric to $\left(\mathbb{R}^{k} \times N_{0}, d_{\mathbb{R}^{k} \times N_{0}}\right)$. In particular, $\left(M, d_{M}\right)$ is isometric to $\left([0, \infty) \times \mathbb{R}^{k} \times N_{0}, d_{[0, \infty) \times \mathbb{R}^{k} \times N_{0}}\right)$.

Proof. Due to Theorem 1.3, the metric space $\left(M, d_{M}\right)$ is isometric to $\left([0, \infty) \times_{F} \partial M, d_{[0, \infty) \times_{F} \partial M}\right)$. Moreover, for every $x \in \partial M$, the function $f \circ \gamma_{x}$ is constant on $[0, \infty)$; in particular, $\left(M, d_{M}\right)$ is isometric to $\left([0, \infty) \times \partial M, d_{[0, \infty) \times \partial M}\right)$. For every $x \in \partial M$, it holds that Hess $f\left(u_{x}, u_{x}\right)=0$. By Lemma 5.6, we have $\operatorname{Ric}_{\left.f\right|_{\partial M}}^{N-1}=\operatorname{Ric}_{f}^{N}$ on the unit tangent bundle over $\partial M$. It follows that $\operatorname{Ric}_{\left.f\right|_{\partial M} ^{N}, \partial M}^{N-1} \geq 0$. Note that $N-1$ is smaller than 1 , and $\sup _{x \in \partial M} f(x)$ is finite. Applying Theorem 5.7 to $\partial M$ inductively, we complete the proof.

5.4. Variants of the splitting theorem. We have already known several rigidity results studied in [13] (and [9], [12]) for manifolds with boundary whose boundaries are disconnected. In [33, the author has given generalizations of them in the usual weighted case (see Subsection 6.4 in [33]). We generalize one of them in our setting. 
For $A_{1}, A_{2} \subset M$, we put $d_{M}\left(A_{1}, A_{2}\right):=\inf _{p_{1} \in A_{1}, p_{2} \in A_{2}} d_{M}\left(p_{1}, p_{2}\right)$. The following has been shown in [13] (see Lemma 1.6 in [13]):

Lemma $5.9([13])$. Suppose that $\partial M$ is disconnected. Let $\left\{\partial M_{i}\right\}_{i=1,2, \ldots}$ denote the connected components of $\partial M$. Assume that $\partial M_{1}$ is compact. Put $D:=\inf _{i=2,3, \ldots} d_{M}\left(\partial M_{1}, \partial M_{i}\right)$. Then there exists a connected component $\partial M_{2}$ of $\partial M$ such that $d_{M}\left(\partial M_{1}, \partial M_{2}\right)=D$. Furthermore, for every $i=1,2$ there exists $x_{i} \in \partial M_{i}$ such that $d_{M}\left(x_{1}, x_{2}\right)=D$. The normal minimal geodesic $\gamma:[0, D] \rightarrow M$ from $x_{1}$ to $x_{2}$ is orthogonal to $\partial M$ both at $x_{1}$ and at $x_{2}$, and the restriction $\left.\gamma\right|_{(0, D)}$ lies in $\operatorname{Int} M$.

We prove the following splitting theorem:

Theorem 5.10. Let $M$ be a connected complete Riemannian manifold with boundary, and let $f: M \rightarrow \mathbb{R}$ be a smooth function. Suppose that $\partial M$ is disconnected. Let $\left\{\partial M_{i}\right\}_{i=1,2, \ldots}$ denote the connected components of $\partial M$. Suppose that $\partial M_{1}$ is compact, and put $D:=$ $\inf _{i=2,3, \ldots} d_{M}\left(\partial M_{1}, \partial M_{i}\right)$. Let $\kappa \in \mathbb{R}$ and $\lambda \in \mathbb{R}$ satisfy the subharmoniccondition. For $N \in(-\infty, 1]$ we suppose $\operatorname{Ric}_{f, M}^{N} \geq \kappa$, and $H_{f, \partial M} \geq \lambda$. Then $\left(M, d_{M}\right)$ is isometric to $\left([0, D] \times_{F} \partial M_{1}, d_{[0, D] \times_{F} \partial M_{1}}\right)$. Moreover, if $N \in(-\infty, 1)$, then for every $x \in \partial M_{1}$ the function $f \circ \gamma_{x}$ is constant on $[0, D]$; in particular, $\left(M, d_{M}\right)$ is isometric to $\left([0, D] \times \partial M_{1}, d_{[0, D] \times \partial M_{1}}\right)$.

Proof. By Lemma 5.9, there exists a connected component $\partial M_{2}$ of $\partial M$ such that $d_{M}\left(\partial M_{1}, \partial M_{2}\right)=D$. For each $i=1,2$, let $\rho_{\partial M_{i}}: M \rightarrow \mathbb{R}$ be the distance function from $\partial M_{i}$ defined as $\rho_{\partial M_{i}}(p):=d_{M}\left(p, \partial M_{i}\right)$. Put

$$
\Omega:=\left\{p \in \operatorname{Int} M \mid \rho_{\partial M_{1}}(p)+\rho_{\partial M_{2}}(p)=D\right\} .
$$

Lemma 5.9 implies that $\Omega$ is a non-empty closed subset of Int $M$.

We show that $\Omega$ is open in Int $M$. Take $p \in \Omega$. For each $i=1,2$, there exists a foot point $x_{p, i} \in \partial M_{i}$ on $\partial M_{i}$ of $p$ such that $d_{M}\left(p, x_{p, i}\right)=$ $\rho_{\partial M_{i}}(p)$. From the triangle inequality, we derive $d_{M}\left(x_{p, 1}, x_{p, 2}\right)=D$. The normal minimal geodesic $\gamma:[0, D] \rightarrow M$ from $x_{p, 1}$ to $x_{p, 2}$ is orthogonal to $\partial M$ at $x_{p, 1}$ and at $x_{p, 2}$. Furthermore, $\left.\gamma\right|_{(0, D)}$ lies in Int $M$ and passes through $p$. There exists an open neighborhood $U$ of $p$ such that $U$ is contained in Int $M$ and $\rho_{\partial M_{i}}$ is smooth on $U$. By using Lemma 3.2 , we see $\Delta_{f} \rho_{\partial M_{i}} \geq 0$ on $U$; in particular, $-\left(\rho_{\partial M_{1}}+\rho_{\partial M_{2}}\right)$ is $f$-subharmonic on $U$. Lemma 5.1 implies that $\Omega$ is open in Int $M$.

Since Int $M$ is connected, we have Int $M=\Omega$. For each $x \in \partial M_{1}$, choose an orthonormal basis $\left\{e_{x, i}\right\}_{i=1}^{n-1}$ of $T_{x} \partial M$. Let $\left\{Y_{x, i}\right\}_{i=1}^{n-1}$ be the $\partial M$-Jacobi fields along $\gamma_{x}$ with initial conditions $Y_{x, i}(0)=e_{x, i}$ and $Y_{x, i}^{\prime}(0)=-A_{u_{x}} e_{x, i}$. Using Lemma 3.5, for all $i$ we see $Y_{x, i}=F_{x} E_{x, i}$ on $[0, D]$, where $\left\{E_{x, i}\right\}_{i=1}^{n-1}$ are the parallel vector fields along $\gamma_{x}$ with initial condition $E_{x, i}(0)=e_{x, i}$. Moreover, if $N \in(-\infty, 1)$, then for 
every $x \in \partial M_{1}$ the function $f \circ \gamma_{x}$ is constant on $[0, D]$. Define a map $\Phi:[0, D] \times \partial M_{1} \rightarrow M$ by $\Phi(t, x):=\gamma_{x}(t)$. The map $\Phi$ is a Riemannian isometry with boundary from $[0, D] \times_{F} \partial M_{1}$ to $M$.

Remark 5.2. Wylie [39] has proved the same result as Theorem 5.10 when $\kappa=0$ and $\lambda=0$ (see Theorem 5.1 in [39]).

\section{EigenVAlue Rigidity}

Let $M$ be an $n$-dimensional, connected complete Riemannian manifold with boundary with Riemannian metric $g$, and let $f: M \rightarrow \mathbb{R}$ be a smooth function.

6.1. Lower bounds. In [33], the author has shown the following Picone type inequality proved by Allegretto and Huang [1] in the Euclidean case (see Theorem 1.1 in [1], and Lemma 7.1 in [33]).

Lemma 6.1 ([33]). Let $\varphi$ and $\psi$ be functions on $M$ that are smooth on a domain $U$ in $M$, and satisfy $\varphi>0$ and $\psi \geq 0$ on $U$. Then for all $p \in(1, \infty)$ we have the following inequality on $U$ :

$$
\|\nabla \psi\|^{p} \geq\|\nabla \varphi\|^{p-2} g\left(\nabla\left(\psi^{p} \varphi^{1-p}\right), \nabla \varphi\right) .
$$

Moreover, if the equality in (6.1) holds on $U$, then for some constant $c \neq 0$ we have $\psi=c \varphi$ on $U$.

We now give a proof of the inequality $(1.9)$ in Theorem 1.5 .

Proposition 6.2. Suppose that $M$ is compact. Let $p \in(1, \infty)$. Let $\kappa \in \mathbb{R}$ and $\lambda \in \mathbb{R}$ satisfy the subharmonic-condition. For $N \in(-\infty, 1]$ we suppose $\operatorname{Ric}_{f, M}^{N} \geq \kappa$, and $H_{f, \partial M} \geq \lambda$. For $D \in(0, \infty)$ we assume $D(M, \partial M) \leq D$. Then we have (1.9).

Proof. Let $\varphi_{p, D}:[0, D] \rightarrow \mathbb{R}$ be a function satisfying (1.8) for $\mu=\mu_{p, D}$. We may assume $\left.\varphi_{p, D}\right|_{(0, D]}>0$. Then we see $\left.\varphi_{p, D}^{\prime}\right|_{[0, D)}>0$. Put $\Phi:=\varphi_{p, D} \circ \rho_{\partial M}$. Take a non-negative, non-zero smooth function $\psi$ on $M$ whose support is compact and contained in Int $M$. By Lemma 6.1.

$$
\|\nabla \psi\|^{p} \geq\|\nabla \Phi\|^{p-2} g\left(\nabla\left(\psi^{p} \Phi^{1-p}\right), \nabla \Phi\right)
$$

on Int $M \backslash$ Cut $\partial M$. From (6.2) and Proposition 3.7, we derive

$$
\begin{aligned}
& \int_{M}\|\nabla \psi\|^{p} d m_{f} \geq \int_{M}\|\nabla \Phi\|^{p-2} g\left(\nabla\left(\psi^{p} \Phi^{1-p}\right), \nabla \Phi\right) d m_{f} \\
\geq & \int_{M}\left(\psi^{p} \Phi^{1-p}\right)\left(-\left(\left(\varphi_{p, D}^{\prime}\right)^{p-1}\right)^{\prime} \circ \rho_{\partial M}\right) d m_{f}=\mu_{p, D} \int_{M} \psi^{p} d m_{f} .
\end{aligned}
$$

It follows that $R_{f, p}(\psi) \geq \mu_{p, D}$. Hence, we arrive at 1.9 . 
Remark 6.1. In Proposition 6.2, we assume that there exists a nonnegative, non-zero smooth function $\psi: M \rightarrow \mathbb{R}$ whose support is compact and contained in Int $M$ such that $R_{f, p}(\psi)=\mu_{p, D}$. Then the equality in (6.2) holds on Int $M \backslash$ Cut $\partial M$, and hence for some constant $c \neq 0$ we have $\psi=c \Phi$ on $M$ (see Lemma 6.1). The equality case in (3.11) also happens (see Remark 3.4).

6.2. Equality cases. For a positive number $D \in(0, \infty)$, we put $S_{D}(\partial M):=B_{D}(\partial M) \backslash U_{D}(\partial M)$.

For the proof of Theorem 1.5 , we show the following lemma concerning the $F$-model space introduced in Subsection 1.4;

Lemma 6.3. Suppose that $M$ is compact. let $\kappa \in \mathbb{R}$ and $\lambda \in \mathbb{R}$ satisfy the subharmonic-condition. For $N \in(-\infty, 1]$ we suppose $\operatorname{Ric}_{f, M}^{N} \geq \kappa$, and $H_{f, \partial M} \geq \lambda$. Assume that for some $D \in(0, \infty)$ we have Cut $\partial M=$ $S_{D}(\partial M)$. For each $x \in \partial M$, choose an orthonormal basis $\left\{e_{x, i}\right\}_{i=1}^{n-1}$ of $T_{x} \partial M$, and let $\left\{Y_{x, i}\right\}_{i=1}^{n-1}$ be the $\partial M-J a c o b i$ fields along $\gamma_{x}$ with initial conditions $Y_{x, i}(0)=e_{x, i}$ and $Y_{x, i}^{\prime}(0)=-A_{u_{x}} e_{x, i}$. Assume further that for all $i$ we have $Y_{x, i}=F_{x} E_{x, i}$ on $[0, D]$, where $\left\{E_{x, i}\right\}_{i=1}^{n-1}$ are the parallel vector fields along $\gamma_{x}$ with initial condition $E_{x, i}(0)=e_{x, i}$. Then $\left(M, d_{M}\right)$ is an F-model space.

Proof. First, we assume that $\partial M$ is disconnected. Let $\left\{\partial M_{i}\right\}_{i=1,2, \ldots}$ be the connected components of $\partial M$. Put $D_{1}:=\inf _{i=2,3, \ldots} d_{M}\left(\partial M_{1}, \partial M_{i}\right)$. By Theorem 5.10, there exists a connected component $\partial M_{1}$ such that $\left(M, d_{M}\right)$ is isometric to $\left(\left[0, D_{1}\right] \times_{F} \partial M_{1}, d_{\left[0, D_{1}\right] \times_{F} \partial M_{1}}\right)$. From Cut $\partial M=$ $S_{D}(\partial M)$, it follows that $D(M, \partial M)=D$ and $D_{1}=2 D$, and hence $\left(M, d_{M}\right)$ is an $F$-model space.

Next, we assume that $\partial M$ is connected. By Cut $\partial M=S_{D}(\partial M)$, we have $D(M, \partial M)=D$. From the property of Jacobi fields, $S_{D}(\partial M)$ is a smooth hypersurface in $M$, and every point in $S_{D}(\partial M)$ has two distinct foot points on $\partial M$. For every $x \in \partial M$, the vector $\gamma_{x}^{\prime}(D)$ is orthogonal to $S_{D}(\partial M)$; and hence the number of foot points on $\partial M$ of $\gamma_{x}(D)$ is equal to two. Now, we define an involutive isometry $\sigma: \partial M \rightarrow \partial M$ without fixed points by $\sigma(x):=y$, where $y$ is the foot point on $\partial M$ of $\gamma_{x}(D)$ that is different from $x$. We also define a map $\Phi:[0,2 D] \times \partial M \rightarrow M$ as follows: If $t \in[0, D)$, then $\Phi(t, x):=\gamma_{x}(t)$; if $t \in(D, 2 D]$, then $\Phi(t, x):=\gamma_{\sigma(x)}(2 D-t)$. We see that $\Phi$ is surjective and continuous. For all $x \in \partial M$ and $t \in[0,2 D]$ we have $\Phi(t, x)=$ $\Phi(2 D-t, \sigma(x))$. Since for all $x \in \partial M$ and $i$ we have $Y_{x, i}=F_{x} E_{x, i}$ on $[0, D]$, the map $\left.\Phi\right|_{[0, D)}$ gives an isometry between $\left(U_{D}(\partial M), g\right)$ and the twisted product space $[0, D) \times_{F} \partial M$. Therefore, $M$ is isometric to the quotient space $\left([0,2 D] \times_{F} \partial M\right) / G_{\sigma}$, where $G_{\sigma}$ is the isometry group 
on $[0,2 D] \times_{F} \partial M$ of the identity and the involute isometry $\hat{\sigma}$ defined by $\hat{\sigma}(t, x):=(2 D-t, \sigma(x))$. This implies that $\left(M, d_{M}\right)$ is an $F$-model space. We complete the proof.

Furthermore, we recall the following fact concerning eigenfunctions for the $(f, p)$-Laplacian (see e.g., [33], [37]):

Proposition 6.4. Suppose that $M$ is compact. Let $p \in(1, \infty)$. Then there exists a non-negative, non-zero function $\Psi$ in $W_{0}^{1, p}\left(M, m_{f}\right)$ such that $R_{f, p}(\Psi)=\mu_{f, 1, p}(M)$. Moreover, for some $\alpha \in(0,1)$ the function $\Psi$ is $C^{1, \alpha}$-Hölder continuous on $M$.

Now, we prove Theorem 1.5 and Corollary 1.6 .

Proof of Theorem 1.5. Suppose that $M$ is compact. Let $p \in(1, \infty)$, and let $\kappa \in \mathbb{R}$ and $\lambda \in \mathbb{R}$ satisfy the subharmonic-condition. For $N \in(-\infty, 1]$ we suppose $\operatorname{Ric}_{f, M}^{N} \geq \kappa$, and $H_{f, \partial M} \geq \lambda$. For $D \in(0, \infty)$, we assume $D(M, \partial M) \leq D$. From Proposition 6.2, we derive (1.9).

We assume that the equality in $(1.9)$ holds. Proposition 6.4 implies that there exists a non-negative, non-zero function $\Psi$ in $W_{0}^{1, p}\left(M, m_{f}\right)$ such that $R_{f, p}(\Psi)=\mu_{p, D}$ and $\Psi$ is $C^{1, \alpha}$-Hölder continuous on $M$. Let $\varphi_{p, D}:[0, D] \rightarrow \mathbb{R}$ be a function satisfying (1.8) for $\mu=\mu_{p, D}$, and let $\left.\varphi_{p, D}\right|_{(0, D]}>0$. Putting $\Phi:=\varphi_{p, D} \circ \rho_{\partial M}$, we see that $\Phi$ coincides with a constant multiplication of $\Psi$ on $M$ (see Remark 6.1); in particular, $\Phi$ is also $C^{1, \alpha}$-Hölder continuous on $M$.

For each $x \in \partial M$, we choose an orthonormal basis $\left\{e_{x, i}\right\}_{i=1}^{n-1}$ of $T_{x} \partial M$. Let $\left\{Y_{x, i}\right\}_{i=1}^{n-1}$ be the $\partial M$-Jacobi fields along $\gamma_{x}$ with initial conditions $Y_{x, i}(0)=e_{x, i}$ and $Y_{x, i}^{\prime}(0)=-A_{u_{x}} e_{x, i}$. Then for all $i$ we have $Y_{x, i}=$ $F_{x} E_{x, i}$ on $[0, \tau(x)]$, where $\left\{E_{x, i}\right\}_{i=1}^{n-1}$ are the parallel vector fields along $\gamma_{x}$ with initial condition $E_{x, i}(0)=e_{x, i}$; moreover, if $N \in(-\infty, 1)$, then $f \circ \gamma_{x}$ is constant on $[0, \tau(x)]$ (see Remark 6.1).

We show Cut $\partial M=S_{D}(\partial M)$. From $D(M, \partial M) \leq D$ we deduce $S_{D}(\partial M) \subset$ Cut $\partial M$. We prove the opposite. Take $p_{0} \in \operatorname{Cut} \partial M$. By the property of Jacobi fields, $\rho_{\partial M}$ is not differentiable at $p_{0}$. From the regularity of $\Phi$, it follows that $\varphi_{p, D}^{\prime}\left(\rho_{\partial M}\left(p_{0}\right)\right)=0$; in particular, $\rho_{\partial M}\left(p_{0}\right)=D$. Hence, Cut $\partial M=S_{D}(\partial M)$. This implies $D(M, \partial M)=$ $D$. By Lemma 6.3, we complete the proof of Theorem 1.5.

Proof of Corollary 1.6. By Theorem 1.5 and Proposition 2.9, we complete the proof of Corollary 1.6.

By Theorem 1.5 and $\mu_{2, D}=\pi^{2}(2 D)^{-2}$, we have the following: 
Corollary 6.5. Let $M$ be a connected complete Riemannian manifold with boundary, and let $f: M \rightarrow \mathbb{R}$ be a smooth function. Suppose that $M$ is compact. Let $\kappa \in \mathbb{R}$ and $\lambda \in \mathbb{R}$ satisfy the subharmonic-condition. For $N \in(-\infty, 1]$ we suppose $\operatorname{Ric}_{f, M}^{N} \geq \kappa$, and suppose $H_{f, \partial M} \geq \lambda$. For $D \in(0, \infty)$ we assume $D(M, \partial M) \leq D$. Then

$$
\mu_{f, 1,2}(M) \geq \frac{\pi^{2}}{4 D^{2}}
$$

If the equality in (6.3) holds, then $D(M, \partial M)=D$, and the metic space $\left(M, d_{M}\right)$ is an $F$-model space. Moreover, if $N \in(-\infty, 1)$, then for every $x \in \partial M$ the function $f \circ \gamma_{x}$ is constant on $[0, D]$; in particular, $\left(M, d_{M}\right)$ is an equational model space.

6.3. Explicit lower bounds. Let $\Omega$ be a relatively compact domain in $M$ such that $\partial \Omega$ is a smooth hypersurface in $M$ satisfying $\partial \Omega \cap \partial M=$ $\emptyset$. For the canonical Riemannian volume measure $\operatorname{vol}_{\partial \Omega}$ on $\partial \Omega$, let $m_{f, \partial \Omega}:=e^{-\left.f\right|_{\partial \Omega}} \operatorname{vol}_{\partial \Omega}$. Put

$$
\delta_{1}(\Omega):=\inf _{p \in \Omega} \rho_{\partial M}(p), \quad \delta_{2}(\Omega):=\sup _{p \in \Omega} \rho_{\partial M}(p) .
$$

In the usual weighted case, the author 33 ] has proved the following volume estimate (see Propositions 8.1 and 8.2 in [33]):

Proposition 6.6 ([33]). Let $M$ be an n-dimensional, connected complete Riemannian manifold with boundary, and let $f: M \rightarrow \mathbb{R}$ be a smooth function. For $N \in[n, \infty]$ we suppose $\operatorname{Ric}_{f, M}^{N} \geq 0$, and $H_{f, \partial M} \geq 0$. Let $\Omega$ be a relatively compact domain in $M$ such that $\partial \Omega$ is a smooth hypersurface in $M$ satisfying $\partial \Omega \cap \partial M=\emptyset$. Then

$$
m_{f}(\Omega) \leq m_{f, \partial \Omega}(\partial \Omega)\left(\delta_{2}(\Omega)-\delta_{1}(\Omega)\right),
$$

where $\delta_{1}(\Omega)$ and $\delta_{2}(\Omega)$ are the values defined as (6.4).

Kasue [14 has obtained Proposition 6.6 in the unweighted case.

In our setting, we have the following volume estimate:

Proposition 6.7. Let $M$ be a connected complete Riemannian manifold with boundary, and let $f: M \rightarrow \mathbb{R}$ be a smooth function. Let $\kappa \in \mathbb{R}$ and $\lambda \in \mathbb{R}$ satisfy the subharmonic-condition. For $N \in(-\infty, 1]$ we suppose $\operatorname{Ric}_{f, M}^{N} \geq \kappa$, and $H_{f, \partial M} \geq \lambda$. Let $\Omega$ be a relatively compact domain in $M$ such that $\partial \Omega$ is a smooth hypersurface in $M$ satisfying $\partial \Omega \cap \partial M=\emptyset$. Then

$$
m_{f}(\Omega) \leq m_{f, \partial \Omega}(\partial \Omega)\left(\delta_{2}(\Omega)-\delta_{1}(\Omega)\right),
$$

where $\delta_{1}(\Omega)$ and $\delta_{2}(\Omega)$ are the values defined as $(6.4)$. 
We can prove Proposition 6.7 only by replacing the role of the comparison result in the usual weighted case with that of Lemma 3.1 in the proof of Proposition 6.6. We omit the proof.

From Proposition 6.6, the author [33] has derived the following estimate in the usual weighted case (see Theorems 8.4 and 8.5 in [33]):

Theorem 6.8 ([33]). Let $M$ be an $n$-dimensional, connected complete Riemannian manifold with boundary, and let $f: M \rightarrow \mathbb{R}$ be a smooth function. Suppose that $\partial M$ is compact. Let $p \in(1, \infty)$. For $N \in[n, \infty]$ we suppose $\operatorname{Ric}_{f, M}^{N} \geq 0$, and $H_{f, \partial M} \geq 0$. For $D \in(0, \infty)$ we assume $D(M, \partial M) \leq D$. Then we have $\mu_{f, 1, p}(M) \geq(p D)^{-p}$.

The author 32 has shown Theorem 6.8 in the unweighted case.

In our setting, we can prove the following result by using Proposition 6.7 instead of Proposition 6.6 in the proof of Theorem 6.8. The argument is in 33 .

Theorem 6.9. Let $M$ be a connected complete Riemannian manifold with boundary, and let $f: M \rightarrow \mathbb{R}$ be a smooth function. Suppose that $\partial M$ is compact. Let $p \in(1, \infty)$, and let $\kappa \in \mathbb{R}$ and $\lambda \in \mathbb{R}$ satisfy the subharmonic-condition. For $N \in(-\infty, 1]$ we suppose $\operatorname{Ric}_{f, M}^{N} \geq \kappa$, and $H_{f, \partial M} \geq \lambda$. For $D \in(0, \infty)$ we assume $D(M, \partial M) \leq D$. Then we have $\mu_{f, 1, p}(M) \geq(p D)^{-p}$.

\section{REFERENCES}

[1] W. Allegretto and Y.X. Huang, A Picone's identity for the p-Laplacian and applications, Nonlinear Anal. 32 (1998), no. 7, 819-830.

[2] D. Bakry and M. Émery, Diffusions hypercontractives, Séminaire de Probabilités, XIX, 1983/84, 177-206, Lecture Notes in Math., 1123, Springer, Berlin, 1985.

[3] V. Bayle, Propriétés de concavité du profil isopérimétrique et applications, $\mathrm{PhD}$ Thesis, Université Joseph-Fourier-Grenoble I, 2003.

[4] A.L. Besse, Einstein Manifolds, Springer-Verlag, Berlin, 1987.

[5] D. Burago, Y. Burago and S. Ivanov, A Course in Metric Geometry, Graduate Studies in Mathematics, 33. American Mathematical Society, Providence, RI, 2001.

[6] E. Calabi, An extension of E. Hopf's maximum principle with an application to Riemannian geometry, Duke Math. J. 25 (1958), 45-56.

[7] J. Cheeger, Degeneration of Riemannian metrics under Ricci curvature bounds, Accademia Nazionale dei Lincei. Scuola Normale Superiore. Lezione Fermiane, 2001.

[8] J. Cheeger and D. Gromoll, The splitting theorem for manifolds of nonnegative Ricci curvature, J. Differential Geom. 6 (1971/72), 119-128.

[9] C. Croke and B. Kleiner, A warped product splitting theorem, Duke Math. J. 67 (1992), no. $3,571-574$. 
[10] F. Fang, X. Li and Z. Zhang, Two generalizations of Cheeger-Gromoll splitting theorem via Bakry-Emery Ricci curvature, Ann. Inst. Fourier (Grenoble) 59 (2009), no. 2, 563-573.

[11] E. Heintze and H. Karcher, A general comparison theorem with applications to volume estimates for submanifolds, Ann. Sci. École Norm. Sup. (4) 11 (1978), no. 4, 451-470.

[12] R. Ichida, Riemannian manifolds with compact boundary, Yokohama Math. J. 29 (1981), no. 2, 169-177.

[13] A. Kasue Ricci curvature, geodesics and some geometric properties of Riemannian manifolds with boundary, J. Math. Soc. Japan 35 (1983), no. 1, 117131.

[14] On a lower bound for the first eigenvalue of the Laplace operator on a Riemannian manifold, Ann. Sci. École Norm. Sup. (4) 17 (1984), no. 1, 31-44.

[15] __ Applications of Laplacian and Hessian Comparison Theorems, Geometry of geodesics and related topics (Tokyo, 1982), 333-386, Adv. Stud. Pure Math., 3, North-Holland, Amsterdam, 1984.

[16] B. Klartag, Needle decompositions in Riemannian geometry, to appear in Mem. Amer. Math. Soc..

[17] A.V. Kolesnikov and E. Milman, Isoperimetric inequalities on weighted manifolds with boundary, Dokl. Akad. Nauk 464 (2015), no. 2, 136-140.

[18] P. Li and S.T. Yau, Estimates of eigenvalues of a compact Riemannian manifold, Proc. Symp. Pure Math. 36 (1980), 205-239.

[19] A. Lichnerowicz, Variétés riemanniennes à tenseur $C$ non négatif, C. R. Acad. Sci. Paris Sér. A-B 271 (1970) A650-A653.

[20] J. Lott, Some geometric properties of the Bakry-Émery-Ricci tensor, Comment. Math. Helv. 78 (2003), no. 4, 865-883.

[21] J. Lott and C. Villani, Weak curvature conditions and functional inequalities, J. Funct. Anal. 245 (2007), no. 1, 311-333.

[22] _ Ricci curvature for metric-measure spaces via optimal transport, Ann. of Math. (2) 169 (2009), no. 3, 903-991.

[23] E. Milman, Beyond traditional Curvature-Dimension I: new model spaces for isoperimetric and concentration inequalities in negative dimension, to appear in Trans. Amer. Math. Soc..

[24] F. Morgan, Manifolds with density, Notices Amer. Math. Soc. 52 (2005), no. 8, 853-858.

[25] S. Ohta, $(K, N)$-convexity and the curvature-dimension condition for negative $N$, J. Geom. Anal. 26 (2016), no. 3, 2067-2096.

[26] S. Ohta and A. Takatsu, Displacement convexity of generalized relative entropies, Adv. Math. 228 (2011), no. 3, 1742-1787.

[27] _ Displacement convexity of generalized relative entropies. II, Comm. Anal. Geom. 21 (2013), no. 4, 687-785.

[28] R. Perales, Volumes and limits of manifolds with Ricci curvature and mean curvature bound, Differential Geom. Appl. 48 (2016), 23-37.

[29] P. Petersen, Riemannian Geometry: Second Edition, Graduate Texts in Mathematics, 171. Springer, New York, 2006.

[30] Z. Qian, Estimates for weighted volumes and applications, Quart. J. Math. Oxford Ser. (2) 48 (1997), no. 190, 235-242. 
[31] T. Sakai, Riemannian Geometry, Translations of Mathematical Monographs, 149. American Mathematical Society, Providence, RI, 1996.

[32] Y. Sakurai, Rigidity of manifolds with boundary under a lower Ricci curvature bound, arXiv preprint arXiv:1404.3845v5 (2015), to appear in Osaka J. Math..

[33] _ _ Rigidity of manifolds with boundary under a lower Bakry-Émery Ricci curvature bound, arXiv preprint arXiv:1506.03223 4 (2016), to appear in Tohoku Math. J..

[34] K.-T. Sturm, On the geometry of metric measure spaces. I, Acta Math. 196 (2006), no. 1, 65-131.

[35] _ On the geometry of metric measure spaces. II, Acta Math. 196 (2006), no. $1,133-177$.

[36] C. Villani, Optimal Transport: Old and New, Springer-Verlag, Berlin, 2009.

[37] P. Tolksdorf, Regularity for a more general class of quasilinear elliptic equations, J. Differential Equations 51 (1984), no. 1, 126-150.

[38] G. Wei and W. Wylie, Comparison geometry for the Bakry-Emery Ricci tensor, J. Differential Geom. 83 (2009), no. 2, 377-405.

[39] W. Wylie, A warped product version of the Cheeger-Gromoll splitting theorem, arXiv preprint arXiv:1506.03800 2 (2016).

[40] W. Wylie and D. Yeroshkin, On the geometry of Riemannian manifolds with density, arXiv preprint arXiv:1602.08000 (2016).

Faculty of Pure and Applied Sciences, University of Tsukuba, TennOdAi 1-1-1, Tsukuba, Ibaraki, 305-8571, Japan

E-mail address: sakurai@math.tsukuba.ac.jp 